TRANSACTIONS OF THE

AMERICAN MATHEMATICAL SOCIETY

Volume 357, Number 12, Pages 4739-4769

S 0002-9947(05)04000-6

Article electronically published on July 19, 2005

\title{
CANONICAL FORMS OF BOREL FUNCTIONS ON THE MILLIKEN SPACE
}

\author{
OLAF KLEIN AND OTMAR SPINAS
}

\begin{abstract}
The goal of this paper is to canonize Borel measurable mappings $\Delta: \Omega^{\omega} \rightarrow \mathbb{R}$, where $\Omega^{\omega}$ is the Milliken space, i.e., the space of all increasing infinite sequences of pairwise disjoint nonempty finite sets of $\omega$. This main result is a common generalization of a theorem of Taylor and a theorem of Prömel and Voigt.
\end{abstract}

\section{INTRODUCTION}

We shall use notation here that will be defined in Section 1 below.

Ramsey's Theorem [Ra30] is an important extension of the pigeonhole principle: If $\omega=P_{0} \cup \cdots \cup P_{k-1}$ is a partition of $\omega$ into finitely many pieces, then for some $i<k, P_{i}$ is infinite.

Theorem 0.1 (Ramsey R). Let $l \in \omega$. If $[\omega]^{l}=P_{0} \cup \cdots \cup P_{k-1}$ is a partition of $[\omega]^{l}$ into finitely many pieces, there is an infinite set $A \in[\omega]^{\omega}$ such that $[A]^{l} \subseteq P_{i}$ for some $i<k$.

Ramsey's Theorem can be viewed as a canonization of finite-range functions on $[\omega]^{l}$. Later P. Erdös and R. Rado ErRa50] canonized arbitrary such functions.

Theorem 0.2 (Erdös-Rado ER). If $k \in \omega$ and $f:[\omega]^{k} \rightarrow \omega$, then there exist an infinite set $X \subseteq \omega$ and a set $\Delta(f, X) \subseteq\{0, \ldots, k-1\}$ such that if $\left\{x_{0}, \ldots, x_{k-1}\right\}$ and $\left\{y_{0}, \ldots, y_{k-1}\right\}$ are in $[X]^{k}$ with $x_{0}<\cdots<x_{k-1}$ and $y_{0}<\cdots<y_{k-1}$, then

$$
f\left(\left\{x_{0}, \ldots, x_{k-1}\right\}\right)=f\left(\left\{y_{0}, \ldots, y_{k-1}\right\}\right) \quad \text { iff } \quad x_{i}=y_{i} \text { for all } i \in \Delta(f, X) .
$$

So abstractly and vaguely, canonizing functions of a given class on a given space means finding a list of canonical functions (the generalized projections in Theorem 0.2 such that an arbitrary function in the given class induces the same equivalence relation as a canonical function on a subdomain that is essentially the same as the original space.

About twenty years later N. Hindman Hi74 analysed the space of all finite subsets of $\omega$. He found the following famous result. For $\kappa \leq \omega$ and $a \in \Omega^{\omega}$ let $(a)^{\kappa}$ denote the collection of all increasing sequences of $\kappa$ pairwise disjoint nonempty finite subsets of $\omega$, which are obtained by unions of some $a(i), i \in \omega$.

Theorem 0.3 (Hindman H). Let $k \in \omega$. If $f:[\omega]<\omega \rightarrow k$, then there exists $a \in \Omega^{\omega}$ such that $f$ is constant on $(a)^{1}$.

Received by the editors March 12, 2002.

2000 Mathematics Subject Classification. Primary 03E15, 05D10, 54H05.

The second author was partially supported by DFG grant SP 683.

(C)2005 American Mathematical Society Reverts to public domain 28 years from publication 
This theorem was the basis of the work of K. R. Milliken and A. D. Taylor mentioned below. Taylor proved a canonical partition relation for finite subsets of $\omega$ that generalizes Hindman's Theorem in much the same way that the Erdös-Rado Theorem generalizes Ramsey's Theorem. In his proof Taylor used an $n$-dimensional version of Theorem 0.3 (denoted $\mathrm{H}^{n}$ below), which was obtained independently also by Milliken (see Lemma 2.5).

The following result of Taylor Ta76 stimulated a part of this work.

Theorem 0.4 (Taylor T). If $f:[\omega]^{<\omega} \rightarrow \omega$, then there exists $a \in \Omega^{\omega}$ such that exactly one of (a)-(e) holds:

(a) If $m, n \in(a)^{1}$, then $f(m)=f(n)$.

(b) If $m, n \in(a)^{1}$, then $f(m)=f(n)$ iff $\min (m)=\min (n)$.

(c) If $m, n \in(a)^{1}$, then $f(m)=f(n)$ iff $\max (m)=\max (n)$.

(d) If $m, n \in(a)^{1}$, then $f(m)=f(n)$ iff $\min (m)=\min (n)$ and $\max (m)=$ $\max (n)$.

(e) If $m, n \in(a)^{1}$, then $f(m)=f(n)$ iff $m=n$.

F. Galvin and K. Prikry have shown in GaPr73 that a similar result to Theorem 0.1 is valid for finite partitions of $[\omega]^{\omega}$ - with the restriction that all pieces of the partitions must be Borel.

Theorem 0.5 (Galvin-Prikry GP). Let $k>0$ and $[\omega]^{\omega}=P_{0} \cup \cdots \cup P_{k-1}$, where each $P_{i}$ is Borel. Then there is an infinite set $A \in[\omega]^{\omega}$ and $i<k$ with $[A]^{\omega} \subseteq P_{i}$.

The power set of $\omega$ can be identified with the Cantor space $2^{\omega}$. It can be endowed with the product topology of the discrete topology on $\omega$. It is a well-known fact that this topological space is completely metrizable. Thus, we can interpret the spaces $[\omega]^{l}$ and $[\omega]^{\omega}$ in the theorems above as topological spaces with the relative topology of $[\omega] \leq \omega$. For distinction we call this topology the metric topology of $[\omega] \leq \omega$.

A subset $P \subseteq[\omega]^{\omega}$ is called Ramsey iff there is an infinite set $A \in[\omega]^{\omega}$ such that either $[A]^{\omega} \subseteq P$ or else $[A]^{\omega} \cap P=\varnothing$. By Theorem 0.5 every Borel set is Ramsey.

J. Silver [Si70] extended the result of Galvin-Prikry to analytic sets. Subsequent to Silver's investigation A. Mathias Ma68] obtained a new proof of the same result.

For stronger results E. Ellentuck has introduced a finer topology on $[\omega]^{\omega}$ which is called Ellentuck topology. For any $a \in[\omega]^{<\omega}$ and $A \in[\omega]^{\omega}$ with $a<A$ let $[a, A]^{\omega}=\left\{S \in[\omega]^{\omega}: a \subseteq S \subseteq a \cup A\right\}$. The Ellentuck topology then has as basic open sets all the sets of the form $[a, A]^{\omega}$ for $a<A$. Note that there are continuum many pairwise disjoint ones of them. Clearly the Ellentuck topology is finer than the metric topology.

Call a set $P \subseteq[\omega]^{\omega}$ completely Ramsey iff for every $a<A$ there is $B \in[A]^{\omega}$ with $[a, B]^{\omega} \subseteq P$ or $[a, B]^{\omega} \cap P=\varnothing$. Ellentuck El74] has shown the following main result, which implies the theorem of Galvin-Prikry.

Theorem 0.6 (Ellentuck). Let $P \subseteq[\omega]^{\omega}$. Then $P$ is completely Ramsey, if $P$ has the Baire property in the Ellentuck topology.

Moreover Galvin [El74] made the observation that every completely Ramsey set has the Baire property. Therewith also the converse of Theorem 0.6 holds. An analogous result with respect to a finer topology - the $\Sigma$-topology — was proven by Milliken (see Theorem 4.4 in [Mi75]). In particular, we take notice of a corollary of Milliken's result (M): Let $k>0$ and let $\Omega^{\omega}=P_{0} \cup \cdots \cup P_{k-1}$ be a partition of 
$\Omega^{\omega}$ into finitely many pieces, where each $P_{i}$ is Borel. Then there exists $a \in \Omega^{\omega}$ and $i<k$ with $(a)^{\omega} \subseteq P_{i}$.

P. Pudlák and V. Rödl PuRö82 canonized Borel-measurable mappings on $[\omega]^{\omega}$ with a countable range. The following result of H. J. Prömel and B. Voigt PrVo85, gives the canonization of such functions with arbitrary range.

Theorem 0.7 (Prömel-Voigt PV). Let $\Delta:[\omega]^{\omega} \rightarrow \mathbb{R}$ be a Borel-measurable mapping. Then there exists $A \in[\omega]^{\omega}$ and there exists $\gamma:[A]^{<\omega} \rightarrow\{s, m\}$ such that the mapping $\Gamma:[A]^{\omega} \rightarrow[A] \leq \omega$ with $\Gamma(X)=\{k \in X: \gamma(X \cap k)=s\}$ has the following properties:

(a) $\Gamma(X) \subseteq X$ for all $X \in[A]^{\omega}$,

(b) for no $X, Y \in[A]^{\omega}$, there exists $k \in \Gamma(Y)$ such that $\Gamma(X)=\Gamma(Y) \cap k$, i.e., no $\Gamma(X)$ is a proper initial segment of some $\Gamma(Y)$,

(c) for all $X, Y \in[A]^{\omega}$, it follows that $\Delta(X)=\Delta(Y)$ iff $\Gamma(X)=\Gamma(Y)$.

The methods of proof of Theorem 0.7 are either taken from or based on the fundamental work of Mathias Ma77. Mathias himself had already proved a canonization result for a much larger class of functions $f:[\omega] \leq \omega \rightarrow[\omega] \leq \omega$ than just the Borel maps (see [Ma77, Theorem 6.1]). It is possible to derive Theorem 0.7 from Mathias' result with some extra work.

Figure 0.1 shows the relation between the theorems mentioned above. Here $A \rightarrow B$ means that $A$ generalizes $B$.

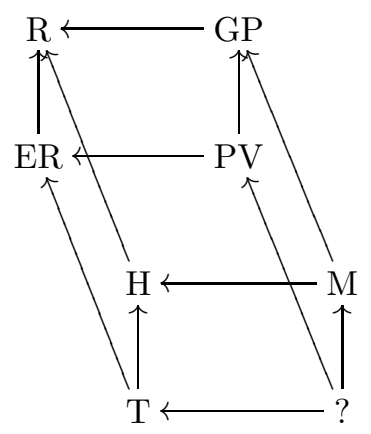

FIGURE 0.1.

All of these implications are pretty obvious and well known. It was natural to search for a theorem that can stand at the place of the interrogation sign. The purpose of this paper is to provide such a theorem.

First, we give some definitions to be able to formulate our Main Theorem. Let $\Omega^{<\omega}$ denote the space of all increasing finite sequences of pairwise disjoint nonempty finite subsets of $\omega$.

Definition. Let $\gamma: \Omega^{<\omega} \rightarrow\{s m$, min-sep, max-sep, min-max, sss, vss $\}$. For $m \in$ $[\omega]^{<\omega}$ let $\operatorname{sm}(m)=\varnothing, \min -\operatorname{sep}(m)=\{\min (m)\}, \max -\operatorname{sep}(m)=\{\max (m)\}$, $\min -\max (m)=\{\min (m), \max (m)\}$ and $s s s(m)=v s s(m)=m$.

Let $x \in \Omega^{\omega}$. Define $\Gamma_{\gamma}(x)$ as follows: Let $k(0)=0$ and $\langle k(i): 0<i<N \leq \omega\rangle$ increasingly enumerate those $k$ such that $\gamma(x 1(k-1))=$ vss. Moreover let $k(N)=\omega$, if $N<\omega$. Now let $\Gamma_{\gamma}(x)=\left\langle\bigcup_{k(i) \leq j<k(i+1)} \gamma(x 1 j)(x(j)): i<N\right\rangle$. 
Now we give our main result:

Main Theorem (MT). For every Borel measurable mapping $\Delta: \Omega^{\omega} \rightarrow \mathbb{R}$ there exist $\gamma: \Omega^{<\omega} \rightarrow\{$ sm, min-sep, max-sep, min-max, sss, vss $\}$ and $a \in \Omega^{\omega}$ such that for all $x, y \in(a)^{\omega}$

$$
\Delta(x)=\Delta(y) \quad \text { iff } \quad \Gamma_{\gamma}(x)=\Gamma_{\gamma}(y) .
$$

Remark. Moreover for the guaranteed $a \in \Omega^{\omega}$ it even holds that for no $x, y \in(a)^{\omega}$ the set $\Gamma_{\gamma}(x)$ is a proper initial segment of $\Gamma_{\gamma}(y)$ (see Lemma 2.36).

We give the proof of the Main Theorem in Section 2, In Section 1 we show that every analytic subset of the Milliken space is completely H-Ramsey-a property that will be used in Section 2, For the implications $\mathrm{MT} \rightarrow \mathrm{T}$ and $\mathrm{MT} \rightarrow \mathrm{PV}$ see the appendix. The implication MT $\rightarrow \mathrm{M}$ is obvious.

In Sp01, the second author has given the canonization of Borel mappings $f:\left(\omega^{\omega}\right)^{2} \rightarrow \mathbb{R}$ modulo restrictions to superperfect rectangles (i.e. rectangles $S_{0} \times S_{1}$ where each $S_{i}$ is closed and homeomorphic to $\omega^{\omega}$ ). Exploiting a link between Milliken neighbourhoods and superperfect rectangles discovered by Todorcevic and Velickovic he noticed that the results of [Sp01] can be derived from the Main Theorem above. This will be presented in a forthcoming note.

\section{The Milliken space}

Hindman's Theorem can be stated in equivalent form speaking about integers and their sums rather than finite sets and their unions. This was first mentioned by Graham and Rothschild GrRo71]. The sum of two integers written in binary notation looks like the characteristic function of the union of two sets, provided the integers in binary are sufficiently spread out so that no carrying occurs upon addition. But the proof of Hindman's Theorem shows that the integers can be chosen with such a property. Milliken [Mi75] stated and proved his results in the sum notation.

The following results up to 1.15 (except for Hindman's Theorem) are essentially Milliken's results in the finite set notation, except for a difference between his notation of $\Sigma$-Ramseyness and our notation of H-Ramseyness. Also see [To98] for an axiomatic treatment of these arguments. First of all let us expand our notation.

We begin by establishing some notation. The set of nonnegative integers is denoted by $\omega$, and we identify each element of $\omega$ with the set of its predecessors as usual, for instance $k=\{0, \ldots, k-1\}$. For the set of all subsets of $X$ which have the same cardinality $k<\omega$ we write $[X]^{k}$. The collection of all finite, resp. countably infinite, subsets of $X$ is denoted by $[X]^{<\omega}$, resp. $[X]^{\omega}$. Moreover let $[X]^{\leq \omega}=[X]^{<\omega} \cup[X]^{\omega}$. If $A \in[\omega]^{<\omega}$ and $B \in[\omega]^{\leq \omega}$, then we write $A<B$ iff both $A$ and $B$ are nonempty and $\max (A)<\min (B)$. Finally, if $s$ is a mapping, we will write $\operatorname{dom}(s)$ to denote the domain of $s$ and $\operatorname{ran}(s)$ to denote the range of $s$.

Definition. Let $\Omega^{\omega}$ denote the collection of all mappings $x: \omega \rightarrow[\omega]<\omega$ with $x(i)<$ $x(j)$ for all $i<j$. If $k \in \omega$, let $\Omega^{k}$ denote all mappings $s: k \rightarrow[\omega]^{<\omega}$ with the same property. Let $\Omega^{<\omega}=\bigcup_{i \in \omega} \Omega^{i}$ and $\Omega^{\leq \omega}=\Omega^{<\omega} \cup \Omega^{\omega}$. Moreover we define $\omega_{\max }$ to be the mapping $\omega \rightarrow[\omega]^{<\omega}$ with $i \mapsto\{i\}$ for every $i \in \omega$.

Definition. If $a \in \Omega^{\omega}$, let $(a)^{\omega}$ denote the set of all mappings $x \in \Omega^{\omega}$ such that for every $i \in \omega$ there exists an $A \in[\omega]^{<\omega}$ with $x(i)=\bigcup_{j \in A} a(j)$. Analogously, if $k \in \omega$, define $(a)^{k}$ to be the set of all mappings $s \in \Omega^{k}$ with the property that for 
every $i \in \operatorname{dom}(s)$ there exists an $A \in[\omega]^{<\omega}$ with $s(i)=\bigcup_{j \in A} a(j)$. Additionally, let $(a)^{<\omega}=\bigcup_{i \in \omega}(a)^{i}$. Finally, if $s \in \Omega^{<\omega}$ and $a \in \Omega^{\omega}$, we use $(s, a)^{\omega}$ to denote the set of mappings $x \in \Omega^{\omega}$ such that $x(i)=s(i)$ for every $i \in \operatorname{dom}(s)$ and for some $b \in(a)^{\omega}, x(i+\operatorname{dom}(s))=b(i)$ for every $i \in \omega$.

Assume that $s, t \in \Omega^{<\omega}$ and $a, b \in \Omega^{\omega}$. We abbreviate $s \in(t)^{<\omega}$, resp. $s \in(b)^{<\omega}$, resp. $a \in(b)^{\omega}$, as $s \ll t$, resp. $s \ll b$, resp. $a \ll b$.

Now let $s \in \Omega^{<\omega}$ and $t \in \Omega^{\leq \omega}$. We write $s<t$ iff $s(\operatorname{dom}(s)-1)<t(0)$, whenever $s$ and $t$ are nonempty. If $s<t$, then we use $s^{\wedge} t$ to denote the mapping $\langle s(i): i<\operatorname{dom}(s), t(i): i<\operatorname{dom}(t)\rangle$. Moreover for every $k \in \omega$ let $t 1 k$ denote the mapping $\langle t(i): i<k\rangle$ and let $t\lceil k$ denote the mapping $\langle t(i): i \geq k\rangle$.

Note that $(a)^{\omega}$ and $[a]^{\omega}, t \uparrow k$ and $t\lceil k$ as well as $s \ll t$ and $s<t$ have different meanings. Regard $\Omega^{\omega}$ as a topological space endowed with the neighbourhood system consisting of sets of the form $(s, a)^{\omega}$ where $s \in \Omega^{<\omega}$ and $a \in \Omega^{\omega}$. We will call $\Omega^{\omega}$ the Milliken space and its topology the $H$-Ellentuck topology. The following results will refer to this topology until we revoke it.

Finally, we want to establish some abbreviations for simpler notation. If $p$ is a mapping with $\operatorname{ran}(p) \leq 1$, we will write $p$ instead of $p(0)$ or $\varnothing$.

For the remainder of this paper let the lower case letters $m, n$ be elements of $\Omega^{1}$, let $p, q$ be elements of $\Omega^{\leq 1}$, let $r, s, t$ be elements of $\Omega^{<\omega}$, let $a, b, c, x, y, z$ be elements of $\Omega^{\omega}$ and let $i, j, k, l$ be elements of $\omega$. Furthermore, let indexed letters be elements of the same space as the corresponding nonindexed letters. Moreover we stipulate that, whenever we write a concatenation like $s^{\wedge} m$, we have $s<m$.

Definition. Let $R \subseteq \Omega^{\omega}$. We say a accepts $s$ iff $(s, a)^{\omega} \subseteq R$ and a rejects $s$ iff there is no $b \ll a$ which accepts $s$. Moreover we say $a$ decides $s$ iff $a$ accepts $s$ or $a$ rejects $s$.

For the next few proofs let $R$ be an arbitrary but fixed set.

Lemma 1.1. There exists a which decides every $s \ll a$.

Proof. Inductively, we construct $a_{j} \in \Omega^{\omega}$ for every $j<\omega$. By definition there is an $a_{0}$ such that $a_{0}$ decides $\varnothing$. Assume that $a_{0}, \ldots, a_{j}$ have been constructed with the property that for every $i \leq j a_{i}$ decides every $s \ll\left\langle a_{0}(0), \ldots, a_{j-1}(0)\right\rangle$. After $2^{j}$ steps we can find an $a_{j+1} \ll a_{j}\left\lceil 1\right.$ which decides every $s \ll\left\langle a_{0}(0), \ldots, a_{j}(0)\right\rangle$. Then $a=\left\langle a_{j}(0): j \in \omega\right\rangle$ has the desired property.

Now we repeat the result of Hindman as Theorem 1.2

Theorem 1.2 (Hindman [Hi74]). If $f:[\omega]^{<\omega} \rightarrow k$, then there exists a such that $f$ is constant on $(a)^{1}$.

For a simpler proof also see [Ba74].

Lemma 1.3. If a decides every $s \ll a$ and a rejects $\varnothing$, then there is $b \ll a$ which rejects every $s \ll b$.

Proof. Inductively, we construct $b_{j} \in \Omega^{\omega}$ for every $j<\omega$. We begin by claiming that there is $b_{0} \ll a$ such that $b_{0}$ rejects every $s \ll b_{0}$ with $\operatorname{dom}(s)=1$. To see this we define a mapping $d:(a)^{1} \mapsto\{a c c, r e j\}$ by $d(s)=a c c$ iff $a$ accepts $s$. By using $f\left(\left\{x_{0}, \ldots, x_{k}\right\}\right)=d\left(\left\langle a\left(x_{0}\right) \cup \cdots \cup a\left(x_{k}\right)\right\rangle\right)$ Theorem 1.2 guarantees the existence of $b_{0} \ll a$ such that $d$ is constant on $\left(b_{0}\right)^{1}$. Now assume that $b_{0}$ accepts every $s \ll b_{0}$ 
with $\operatorname{dom}(s)=1$. Thus, $\left(s, b_{0}\right)^{\omega} \subseteq R$ for every $s \in\left(b_{0}\right)^{1}$. But then $\left(b_{0}\right)^{\omega} \subseteq R$, as $\left(b_{0}\right)^{\omega}=\bigcup\left\{\left(s, b_{0}\right)^{\omega}: s \in\left(b_{0}\right)^{1}\right\}$, contradicting that $a$ rejects $\varnothing$.

For the inductive step, using the same arguments repeatedly we can construct $b_{j} \ll b_{j-1}\left\lceil 1\right.$ such that $b_{j}$ rejects all $s \ll b_{j}$ with $\operatorname{dom}(s)=j+1$. The assertion follows by putting $b=\left\langle b_{j}(0): j \in \omega\right\rangle$.

Definition. We call $R \subseteq \Omega^{\omega} H$-Ramsey iff there is $a$ such that $(\varnothing, a)^{\omega} \subseteq R$ or $(\varnothing, a)^{\omega} \subseteq \Omega^{\omega} \backslash R$.

Lemma 1.4. Every open set $R \subseteq \Omega^{\omega}$ is H-Ramsey.

Proof. Take an $a$ which by Lemma 1.1 decides every $s \ll a$. If $a$ accepts $\varnothing$, then $(a)^{\omega} \subseteq R$. Otherwise $a$ rejects $\varnothing$, and by Lemma 1.3 we may assume that $a$ rejects every $s \ll a$. Suppose that $b \in(a)^{\omega} \cap R$. Then there is a neighbourhood $(r, c)^{\omega}$ such that $b \in(r, c)^{\omega} \subseteq R$. This implies that $b$ accepts $r$ and thus $a$ cannot reject $r$, a contradiction.

The following two lemmas were obtained by straightforward generalizations of the proofs of Lemmas 1.1 and 1.3 .

Lemma 1.5. For every $s$ and $a$ there exists $b \ll a$ such that $b$ decides $s^{\wedge} t$ for every $t \ll b$.

Lemma 1.6. If a decides $s^{\wedge} t$ for every $t \ll a$ and a rejects $s$, then there is $b \ll a$ which rejects $s^{\wedge} t$ for every $t \ll b$.

Definition. We call $C \subseteq \Omega^{\omega}$ completely $H$-Ramsey iff for every $s$ and $a$ there is $b \ll a$ such that $(s, b)^{\omega} \subseteq C$ or $(s, b)^{\omega} \subseteq \Omega^{\omega} \backslash C$.

The following lemma follows from Lemmas 1.5 and 1.6 as Lemma 1.4 did from 1.1 and 1.3 .

Lemma 1.7. Every open set $C \subseteq \Omega^{\omega}$ is completely H-Ramsey.

Corollary 1.8. The complement of a completely H-Ramsey set is completely HRamsey.

Proof. Obvious from the definition.

Definition. We call $C \subseteq \Omega^{\omega} H$-Ramsey null iff for every $s$ and $a$ there is $b \ll a$ such that $(s, b)^{\omega} \subseteq \Omega^{\omega} \backslash C$.

Lemma 1.9. If $C \subseteq \Omega^{\omega}$ is nowhere dense, then it is H-Ramsey null.

Proof. By Lemma 1.7 and Corollary 1.8 the closure $\bar{C}$ of $C$ is completely H-Ramsey. Then there is $b \ll a$ such that $(s, b)^{\omega} \subseteq \bar{C}$ or $(s, b)^{\omega} \subseteq \Omega^{\omega} \backslash \bar{C} \subseteq \Omega^{\omega} \backslash C$. Since $\bar{C}$ is nowhere dense, the former case cannot occur.

Lemma 1.10. If $C \subseteq \Omega^{\omega}$ is meager, then it is H-Ramsey null.

Proof. Let $C_{n}$ be a sequence of nowhere dense sets whose union is $C$. We may assume that $C_{n} \subseteq C_{n+1}$ holds for all $n$.

Inductively, we construct $b_{j} \in \Omega^{\omega}$ for every $j<\omega$. For any $s$ and $a$, by Lemma 1.9 we can get $b_{0} \ll a$ such that $\left(s, b_{0}\right)^{\omega} \subseteq \Omega^{\omega} \backslash C_{0}$. Assume that $b_{0}, \ldots, b_{j}$ have been constructed such that $\left(s^{\wedge} t, b_{j}\right)^{\omega} \subseteq \Omega^{\omega} \backslash C_{j}$ for every $t \ll\left\langle b_{0}(0), \ldots, b_{j-1}(0)\right\rangle$. Then also by Lemma 1.9 we can find $b_{j+1} \ll b_{j}\left\lceil 1\right.$ such that $\left(s^{\wedge} t, b_{j+1}\right)^{\omega} \subseteq \Omega^{\omega} \backslash C_{j+1}$ for every $t \ll\left\langle b_{0}(0), \ldots, b_{j}(0)\right\rangle$.

Hence $b=\left\langle b_{j}(0): j \in \omega\right\rangle$ satisfies the assertion of the lemma. 
Corollary 1.11. Every subset of $\Omega^{\omega}$ is nowhere dense iff it is meager iff it is H-Ramsey null.

Proof. Obvious from the definition of nowhere dense sets and Lemma 1.10.

Lemma 1.12. Every set $C \subseteq \Omega^{\omega}$ with the Baire property is completely H-Ramsey.

Proof. Any set $C$ with the Baire property can be expressed as $C=C_{0} \triangle C_{1}$ where $C_{0}$ is open, $C_{1}$ is meager and $\triangle$ is the symmetric difference. Since $C_{1}$ is meager, by Lemma 1.10 for any $s$ and $a$ we can choose $b \ll a$ so that $(s, b)^{\omega} \subseteq \Omega^{\omega} \backslash C_{1}$ and then by Lemma 1.7 there is $c \ll b$ such that $(s, c)^{\omega} \subseteq C_{0}$ or $(s, c)^{\omega} \subseteq \Omega^{\omega} \backslash C_{0}$. In the former case $(s, c)^{\omega} \subseteq C$ and in the latter $(s, c)^{\omega} \subseteq \Omega^{\omega} \backslash C$.

Lemma 1.13. Let $C \subseteq \Omega^{\omega}$. Then $C$ is completely H-Ramsey iff $C$ has the Baire property.

Proof. Let $C$ be completely H-Ramsey. Then we claim that $N=C \backslash \operatorname{Int}(C)$ is nowhere dense (so $C$ has the Baire property). Indeed, if this fails, there are $s$ and $a$ such that $(s, a)^{\omega} \subseteq \bar{N}$. Let $b \ll a$ be such that $(s, b)^{\omega} \subseteq C$ or $(s, b)^{\omega} \subseteq \Omega^{\omega} \backslash C$. Since $(s, b)^{\omega} \cap N \neq \varnothing,(s, b)^{\omega} \subseteq \Omega^{\omega} \backslash C$ is impossible. So $(s, b)^{\omega} \subseteq C$, thus $(s, b)^{\omega} \subseteq \operatorname{Int}(C)$ and $(s, b)^{\omega} \cap N=\varnothing$, giving a contradiction.

Hence, the assertion of the lemma follows by Lemma 1.12

A Souslin system is a class of closed sets that are indexed by finite sequences of nonnegative integers. A Souslin set is one which can be expressed in the form $\bigcup_{f \in \omega^{\omega}} \bigcap_{k \in \omega} S_{f 1 k}$ where $\left\{S_{e}\right\}_{e}$ is a Souslin system, $f 1 k$ is the restriction of $f$ to the predecessors of $k$, and $\omega^{\omega}$ is the set of all functions mapping $\omega$ into $\omega$.

Lemma 1.14. Every Souslin set $C \subseteq \Omega^{\omega}$ is completely H-Ramsey.

Proof. The Baire property is preserved under the Souslin operation; for a proof see Ku66. Since closed sets have the Baire property, Lemma 1.12 gives our result.

The following result and also the results of the remainder of this paper will refer to the metric topology on $\Omega^{\omega}$. Note that by definition the Milliken space is a subspace of $\left([\omega]^{<\omega}\right)^{\omega}$. The latter can be regarded as a topological space with the product topology of the discrete topology of $[\omega]^{<\omega}$. Hence the metric topology on $\Omega^{\omega}$ is the relative topology on $\left([\omega]^{<\omega}\right)^{\omega}$. Notice that it is completely metrizable and coarser than the H-Ellentuck topology.

Theorem 1.15. Every analytic set $C \subseteq \Omega^{\omega}$ is completely H-Ramsey.

Proof. Every analytic set is a Souslin set Ku66. Since closed sets in the metric topology on $\Omega^{\omega}$ are also closed in the H-Ellentuck topology, Lemma 1.14 applies directly.

\section{Proof of the Main Theorem}

The proof of the Main Theorem requires some further results. Our first lemma is analogous to Lemma 1 in [PrVo85.

Lemma 2.1. Let $\Delta: \Omega^{\omega} \rightarrow \mathbb{R}$ be Borel-measurable. Then there exists a such that the restriction $\Delta 1(a)^{\omega}$ is a continuous mapping. 
Proof. Let $\left(I_{j}\right)_{j \in \omega}$ be an enumeration of all open intervals in $\mathbb{R}$ which have rational endpoints. The $I_{j}$ form a basis for the topology of the reals. Inductively, we construct $a_{j} \in \Omega^{\omega}$ for every $j<\omega$. Put $a_{0}=\omega_{\max }$ and assume by induction that $a_{0}, \ldots, a_{j}$ have been constructed such that for all $i<j$ and all $s \ll\left\langle a_{0}(0), \ldots, a_{i}(0)\right\rangle$ either $\left(s, a_{i+1}\right)^{\omega} \subseteq \Delta^{-1}\left(I_{i}\right)$ or $\left(s, a_{i+1}\right)^{\omega} \subseteq \Omega^{\omega} \backslash \Delta^{-1}\left(I_{i}\right)$. Since $I_{j}$ is open, it follows that $\Delta^{-1}\left(I_{j}\right) \subseteq \Omega^{\omega}$ must be Borel and hence by Theorem 1.15 completely HRamsey. Hence we can get an $a_{j+1} \ll a_{j}\left\lceil 1\right.$ such that for all $s \ll\left\langle a_{0}(0), \ldots, a_{j}(0)\right\rangle$ either $\left(s, a_{j+1}\right)^{\omega} \subseteq \Delta^{-1}\left(I_{j}\right)$ or $\left(s, a_{j+1}\right)^{\omega} \subseteq \Omega^{\omega} \backslash \Delta^{-1}\left(I_{j}\right)$.

We claim that $a=\left\langle a_{j}(0): j \in \omega\right\rangle$ has the desired properties. To see this we shall prove that every inverse image $\left(\Delta 1(a)^{\omega}\right)^{-1}\left(I_{j}\right)$ is the union of all open sets $(s, a)^{\omega}$ with the property that $s \ll\left\langle a_{i}(0): i \leq j\right\rangle$ and $(s, a)^{\omega} \subseteq \Delta^{-1}\left(I_{j}\right)$. It is obvious that the union of these open sets is part of the inverse image. So let $x$ be an arbitrary but fixed element of the inverse image. Therefore $x \ll a$ and $\Delta(x) \in I_{j}$. Let $k$ be maximal such that $s=x 1 k \ll\left\langle a_{i}(0): i \leq j\right\rangle$. Then it follows that $x \in(s, a)^{\omega} \subseteq\left(\Delta 1(a)^{\omega}\right)^{-1}\left(I_{j}\right)$ and, thus, the assertion of the lemma.

Remark. Suppose $\Delta: \Omega^{\omega} \rightarrow \mathbb{R}$ is Baire measurable with respect to the H-Ellentuck topology. The same argument, using Lemma 1.12 instead of Theorem 1.15] shows that $\Delta 1(a)^{\omega}$ is continuous with respect to the metric topology on $\Omega^{\omega}$ for some $a$.

For the remainder of this section let $\Delta: \Omega^{\omega} \rightarrow \mathbb{R}$ be an arbitrary but fixed mapping.

Definition. Let $s, t$ and $x$ be such that $s<t, x$ and $s=\langle s(0), \ldots, s(k)\rangle$. We abbreviate the mappings $s 1 k^{\wedge}\langle s(k) \cup t(0)\rangle^{\wedge} t\left\lceil 1\right.$, resp. $s 1 k^{\wedge}\langle s(k) \cup x(0)\rangle^{\wedge} x\lceil 1$,

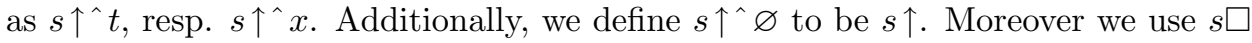
as a variable for $s$ or $s \uparrow$.

Analogously to PrVo85, we introduce now the terms separating and mixing.

Definition. We say that $s \square$ and $t \square$ are separated by $a$ iff $\Delta\left(s \square^{\wedge} x\right) \neq \Delta\left(t \square^{\wedge} y\right)$ for all $x, y \ll a$ with $s<x, y$ and $t<x, y$. Moreover $s \square$ and $t \square$ are mixed by a iff for no $b \ll a$ the sets $s \square$ and $t \square$ are separated by $b$. Finally, $s \square$ and $t \square$ are decided by $a$ iff $s \square$ and $t \square$ are separated or mixed by $a$.

We stipulate that, whenever we write a concatenation like $s \square^{\wedge} m \square$, resp. $s \square^{\wedge}$ $m \square^{\wedge} n \square$, we have $s<m$, resp. $s<m<n$.

Corollary 2.2. For every $s, t$ and $a$, there exists $b \ll a$ which decides $s \square$ and $t \square$. If $s \square$ and $t \square$ are decided by $b$, then they are also decided by each $c \ll b$, and $c$ decides in the same way as $b$ does.

Proof. Obvious from the definition.

Lemma 2.3 (Transitivity of mixing). Assume that $r \square$ and $s \square$ as well as $s \square$ and $t \square$ are mixed by a. Then also $r \square$ and $t \square$ are mixed by $a$.

Proof. Assume to the contrary that there exists $b \ll a$ which separates $r \square$ and $t \square$. We may assume without loss of generality that $r, s, t<b$. Consider the set $A=\left\{x \ll b: \exists y \ll b \Delta\left(r \square^{\wedge} y\right)=\Delta\left(s \square^{\wedge} x\right)\right\}$. Then $A$ is analytic, so by Theorem 1.15 $A$ is completely H-Ramsey. By definition of completely H-Ramsey there exists $c \ll b$ with $(c)^{\omega} \subseteq A$ or $(c)^{\omega} \cap A=\varnothing$. Both cases lead to a contradiction:

Assume first that $(c)^{\omega} \subseteq A$. Then for all $x \ll c$ there exists $y \ll b$ such that $\Delta\left(r \square^{\wedge} y\right)=\Delta\left(s \square^{\wedge} x\right)$. Since $r \square$ and $t \square$ are separated by $b$, it follows that 
$\Delta\left(r \square^{\wedge} y\right) \neq \Delta\left(t \square^{\wedge} z\right)$ for every $y, z \ll b$. Hence we get $\Delta\left(s \square^{\wedge} x\right) \neq \Delta\left(t \square^{\wedge} z\right)$ for all $x, z \ll c$, contradicting that $s \square$ and $t \square$ are mixed by $a$.

Otherwise if $(c)^{\omega} \cap A=\varnothing$, then $r \square$ and $s \square$ are separated by $c$.

Lemma 2.4. For every a there exists $b \ll a$ such that for every $s, t \ll b$ the sets $s \square$ and $t \square$ are decided by $b$.

Proof. Inductively, we construct $b_{j} \in \Omega^{\omega}$ for every $j<\omega$. By Corollary 2.2 there exists $b_{0} \ll a$ such that $\varnothing$ and $\varnothing$ are decided by $b_{0}$. Assume that $b_{0}, \ldots, b_{j}$ have been constructed such that for every $i \leq j$ and for all $s, t \ll\left\langle b_{k}(0): k<i\right\rangle$ the sets $s \square$ and $t \square$ are decided by $b_{i}$. Some applications of Corollary 2.2 yield $b_{j+1} \ll$ $b_{j} \uparrow 1$ such that the inductive assumption is also satisfied for $b_{0}, \ldots, b_{j+1}$. Then $b=\left\langle b_{j}(0): j \in \omega\right\rangle$ has the desired properties.

Lemma 2.5 (Taylor Ta76]). If $k, l>0$ and $f:(a)^{l} \rightarrow k$, then there exists $b \ll a$ such that $f$ is constant on $(b)^{l}$.

For a proof see Lemma 2.2 in [Ta76].

The following lemma is modeled on the image of Theorem 2.1 in Ta76].

Lemma 2.6. For every $s$ and $a$, there exists $b \ll a$ such that exactly one of the following properties holds:

(a) If $m, n \ll b$, then $s^{\wedge} m$ and $s^{\wedge} n$ are mixed by $b$.

(b) If $m, n \ll b$, then $s^{\wedge} m$ and $s^{\wedge} n$ are mixed by $b$ iff $\min (m)=\min (n)$.

(c) If $m, n \ll b$, then $s^{\wedge} m$ and $s^{\wedge} n$ are mixed by $b$ iff $\max (m)=\max (n)$.

(d) If $m, n \ll b$, then $s^{\wedge} m$ and $s^{\wedge} n$ are mixed by $b$ iff $\min (m)=\min (n)$ and $\max (m)=\max (n)$.

(e) If $m, n \ll b$, then $s^{\wedge} m$ and $s^{\wedge} n$ are mixed by $b$ iff $m=n$.

Proof. Lemma 2.4 guarantees the existence of $b_{0} \ll a$ such that $s^{\wedge} m$ and $s^{\wedge} n$ are decided by $b_{0}$ for every $m, n \ll b_{0}$. Let $F$ be the set of all functions $f$ such that $\operatorname{dom}(f)=3$ and $\operatorname{ran}(f) \subseteq 2$. Define $g:\left(b_{0}\right)^{3} \rightarrow F$ as follows:

$g(h)(0)=0$ iff $s^{\wedge}\langle h(0) \cup h(1) \cup h(2)\rangle$ and $s^{\wedge}\langle h(0)\rangle$ are mixed by $b_{0}$.

$g(h)(1)=0$ iff $s^{\wedge}\langle h(0) \cup h(1) \cup h(2)\rangle$ and $s^{\wedge}\langle h(2)\rangle$ are mixed by $b_{0}$.

$g(h)(2)=0$ iff $s^{\wedge}\langle h(0) \cup h(1) \cup h(2)\rangle$ and $s^{\wedge}\langle h(0) \cup h(2)\rangle$ are mixed by $b_{0}$.

By Lemma 2.5 there exists $b_{1} \ll b_{0}$ and a function $f=\langle f(0), f(1), f(2)\rangle \in F$ such that $g\left(\left(b_{1}\right)^{3}\right)=\{f\}$. We claim first that $f$ cannot be $\langle 0,0,1\rangle$ or $\langle 1,0,1\rangle$ or $\langle 0,1,1\rangle$. The first two are ruled out by the observation that if $f(1)=0$, then we must have $f(2)=0$. Indeed, if $f(2) \neq 0$, then $s^{\wedge}\left\langle\left(b_{1}(0) \cup b_{1}(1)\right) \cup b_{1}(2) \cup b_{1}(3)\right\rangle$ and $s^{\wedge}\left\langle\left(b_{1}(0) \cup b_{1}(1)\right) \cup b_{1}(3)\right\rangle$ are separated by $b_{1}$. But since $f(1)=0$, both of these are mixed with $s^{\wedge}\left\langle b_{1}(3)\right\rangle$. By transitivity of mixing we get a contradiction. Similarly, the third one is ruled out since if $f(0)=0$, then we must have $f(2)=0$. This leaves five possibilities for $f$.

We will show that these five possibilities correspond to the five clauses (a)-(e) of this lemma. By construction we are guaranteed that exactly one case holds in the assertion.

Case (a). $f=\langle 0,0,0\rangle$. Let $b=\left\langle b_{1}(i): i>1\right\rangle$ and $m, n \ll b$. Since $f(1)=0$, $s^{\wedge} m$ and $s^{\wedge}\left\langle b_{1}(0) \cup b_{1}(1) \cup m\right\rangle$ as well as $s^{\wedge} n$ and $s^{\wedge}\left\langle b_{1}(0) \cup b_{1}(1) \cup n\right\rangle$ are mixed by $b$. Moreover because $f(0)=0$, we have that $s^{\wedge}\left\langle b_{1}(0) \cup b_{1}(1) \cup m\right\rangle$ and $s^{\wedge}\left\langle b_{1}(0)\right\rangle$ as well as $s^{\wedge}\left\langle b_{1}(0) \cup b_{1}(1) \cup n\right\rangle$ and $s^{\wedge}\left\langle b_{1}(0)\right\rangle$ are mixed by $b$. By transitivity of mixing it follows that $s^{\wedge} m$ and $s^{\wedge} n$ are mixed by $b$ whenever $m, n \ll b$, so $b$ satisfies clause (a) of the lemma. 
Case (b). $f=\langle 0,1,0\rangle$. Let $b=\left\langle b_{1}(3 i) \cup b_{1}(3 i+1) \cup b_{1}(3 i+2): i<\omega\right\rangle$. Suppose first that $m, n \ll b$ with $\min (m)=\min (n)$. Then $m=\langle b(k) \cup p\rangle$ and $n=\langle b(k) \cup q\rangle$ for some $k$ and some $p, q \ll b 1 k$. Since $f(0)=0, s^{\wedge} m$ and $s^{\wedge}\left\langle b_{1}(3 k)\right\rangle$ as well as $s^{\wedge} n$ and $s^{\wedge}\left\langle b_{1}(3 k)\right\rangle$ are mixed by $b$. By transitivity of mixing we obtain that $s^{\wedge} m$ and $s^{\wedge} n$ are mixed by $b$.

Conversely, if $m, n \ll b$ and $\min (m)<\min (n)$, then $m=\langle b(k) \cup p\rangle$ for some $k$ and $p \ll b 1 k$, and $b(k)<n$. Thus, $s^{\wedge} m$ and $s^{\wedge}\langle b(k) \cup n\rangle$ are mixed by $b$, since both are mixed with $s^{\wedge}\left\langle b_{1}(3 k)\right\rangle$ by virtue of the fact that $f(0)=0$. But since $f(1)=1$, we have that $s^{\wedge}\langle b(k) \cup n\rangle$ and $s^{\wedge} n$ are separated by $b$, and so-by transitivity of mixing - we must have that $s^{\wedge} m$ and $s^{\wedge} n$ are separated by $b$.

Thus, $s^{\wedge} m$ and $s^{\wedge} n$ are mixed by $b$ iff $\min (m)=\min (n)$, so $b$ satisfies clause (b) of the lemma.

Case (c). $f=\langle 1,0,0\rangle$. Let $b=\left\langle b_{1}(3 i) \cup b_{1}(3 i+1) \cup b_{1}(3 i+2): i<\omega\right\rangle$ like in case (b). If $m, n \ll b$ and $\max (m)=\max (n)$, then $m=\langle p \cup b(k)\rangle$ and $n=\langle q \cup b(k)\rangle$ for some $k$ and $p, q \ll b 1 k$. Since $f(1)=0$ we have that $s^{\wedge} m$ and $s^{\wedge}\left\langle b_{1}(3 k+2)\right\rangle$ as well as $s^{\wedge} n$ and $s^{\wedge}\left\langle b_{1}(3 k+2)\right\rangle$ are mixed by $b$. Hence $s^{\wedge} m$ and $s^{\wedge} n$ are mixed by $b$.

Conversely, if $m, n \ll b$ and $\max (m)<\max (n)$, then $n=\langle q \cup b(k)\rangle$ for some $k$ and $q \ll b 1 k$, and $m<b(k)$. Thus, $s^{\wedge} n$ and $s^{\wedge}\langle m \cup b(k)\rangle$ are mixed by $b$, because both are mixed with $s^{\wedge}\left\langle b_{1}(3 k+2)\right\rangle$ since $f(1)=0$. But $s^{\wedge}\langle m \cup b(k)\rangle$ and $s^{\wedge} m$ are separated by $b$ since $f(0)=1$. So we must have that $s^{\wedge} m$ and $s^{\wedge} n$ are separated by $b$.

Hence $s^{\wedge} m$ and $s^{\wedge} n$ are mixed by $b$ iff $\max (m)=\max (n)$, and hence $b$ satisfies clause (c) of the lemma.

Claim 2.6.1. Let $s$ and $a$ be such that $s^{\wedge} m$ and $s^{\wedge} n$ are decided by a for every $m, n \ll a$. If $s^{\wedge}\langle h(0) \cup h(1) \cup h(2)\rangle$ and $s^{\wedge}\langle h(0)\rangle$ are separated by a for all $h \in(a)^{3}$, then there exists $b \ll a$ such that $s^{\wedge} m$ and $s^{\wedge} n$ are separated by $b$ for every $m, n \ll b$ with $\max (m)<\max (n)$.

Proof. Let $a_{0}, a_{1}$ be elements of $\Omega^{\omega}$. We construct $b$ inductively. Put $b(0)=a(0)$ and suppose that $b(0), \ldots, b(k-1)$ have been constructed such that $s^{\wedge} m$ and $s^{\wedge} n$ are separated by $a$ for all $m, n \ll b 1 k$ with $\max (m)<\max (n)$. Let $a_{0} \ll a$ with $b(k)\left\langle a_{0}(0)\right.$. Choose $\langle b(k)\rangle \ll a_{0}$ such that $s^{\wedge} m$ and $s^{\wedge}\langle p \cup b(k)\rangle$ are separated by $a$ for every $m, p \ll b 1 k$. This is possible, since otherwise for all $\langle b(k)\rangle \ll a_{0}$ there would exist $m, p \ll b 1 k$ such that $s^{\wedge} m$ and $s^{\wedge}\langle p \cup b(k)\rangle$ are mixed by $a$. Hindman's Theorem would yield $a_{1} \ll a_{0}$ and fixed $m, p \ll b 1 k$ such that $s^{\wedge} m$ and $s^{\wedge}\langle p \cup b(k)\rangle$ are mixed by $a$ for every $\langle b(k)\rangle \ll a_{1}$. By transitivity of mixing (Lemma 2.3) we get that $s^{\wedge}\langle p \cup m\rangle$ and $s^{\wedge}\langle p \cup n\rangle$ are mixed by $a$ for all $m, n \ll a_{1}$. Choosing $h=\left\langle p \cup a_{1}(0), a_{1}(1), a_{1}(2)\right\rangle$ we get a contradiction to the assumption of the lemma. This completes the construction of $b$.

Case (d). $f=\langle 1,1,0\rangle$. To handle case (d) we choose $b_{2} \ll b_{1}$ as guaranteed to exist by Claim 2.6.1, Let $b=\left\langle b_{1}(3 i) \cup b_{1}(3 i+1) \cup b_{1}(3 i+2): i<\omega\right\rangle$. We claim that if $m, n \ll b$, then $s^{\wedge} m$ and $s^{\wedge} n$ are mixed by $b$ iff $\min (m)=\min (n)$ and $\max (m)=\max (n)$.

Suppose first that $\min (m)=\min (n)$ and $\max (m)=\max (n)$. Then for some $i<j$ we have that $s^{\wedge} m$ and $s^{\wedge}\left\langle b_{2}(3 i) \cup b_{2}(3 j+2)\right\rangle$ as well as $s^{\wedge} n$ and $s^{\wedge}\left\langle b_{2}(3 i) \cup b_{2}(3 j+2)\right\rangle$ are mixed by $b$, since $f(2)=0$. By transitivity of mixing we get that $s^{\wedge} m$ and $s^{\wedge} n$ are mixed by $b$. 
For the converse, suppose that either $\min (m) \neq \min (n)$ or $\max (m) \neq \max (n)$. If $\max (m) \neq \max (n)$, then clearly $s^{\wedge} m$ and $s^{\wedge} n$ are separated by $b$, by construction according to Claim 2.6.1. Hence we can assume that $\max (m)=\max (n)$ and $\min (m)<\min (n)$. Let $m=\langle b(k) \cup p \cup b(l)\rangle$ for some $k<l$ and some $p \ll\langle b(i): k<i<l\rangle$ and $b(k)<n$. But then $s^{\wedge} m$ and $s^{\wedge}\left\langle b_{2}(3 k) \cup b_{2}(3 l+2)\right\rangle$ as well as $s^{\wedge}\left\langle b_{2}(3 k) \cup b_{2}(3 l+2)\right\rangle$ and $s^{\wedge}\langle b(k) \cup n\rangle$ are mixed by $b$, since $f(2)=0$. However, since $f(1)=1$ we have that $s^{\wedge}\langle b(k) \cup n\rangle$ and $s^{\wedge} n$ are separated by $b$, and by the transitivity of mixing it follows that $s^{\wedge} m$ and $s^{\wedge} n$ are separated by $b$. Thus, we have shown that $b$ satisfies clause (d) of the lemma.

Definition. For some given $s$ and $a$ we will say that $t$ and $b$ are compatible iff $t<b$ and $s^{\wedge}\langle p \cup m\rangle$ and $s^{\wedge}\langle q \cup m\rangle$ are separated by $a$ for every $m \ll b$ and for all $p$, $q \ll t$ with $\max (p) \neq \max (q)$. Note that $p$ and $q$ can be empty as agreed in the introduction. We will say that $t$ and $b$ are very compatible iff they are compatible and, moreover, there exists $n \ll b$ and there exists $c \ll b$ such that $t^{\wedge} n$ and $c$ are compatible.

Claim 2.6.2. Let $s$ and $a$ be such that $s^{\wedge} m$ and $s^{\wedge} n$ are decided by a for every $m, n \ll a$. Suppose that $s^{\wedge}\langle h(0) \cup h(1) \cup h(2)\rangle$ and $s^{\wedge}\langle h(0) \cup h(2)\rangle$ are separated by a for all $h \in(a)^{3}$. Then if $t$ and $b$ are compatible where $b, t \ll a$, then $t$ and $b$ are in fact very compatible.

Proof. Suppose that $t$ and $b$ are compatible but not very compatible. Then for every $m \ll b$ and for all $c \ll b$ with $m<c$ there exists $n \ll c$ and there exists $p, q \ll t^{\wedge} m$ such that $\max (p) \neq \max (q)$ and $s^{\wedge}\langle p \cup n\rangle$ and $s^{\wedge}\langle q \cup n\rangle$ are mixed by $a$. Notice that we cannot have both $p, q \ll t$ since $t$ and $b$ are compatible. Thus, we better use instead of any such $q$ a mapping of the form $\langle q \cup m\rangle$ with the restriction $q \ll t$. Now two applications of Lemma 2.5 yield $c \ll b$ and fixed $p$, $q \ll t$ such that $s^{\wedge}\langle p \cup n\rangle$ and $s^{\wedge}\langle q \cup m \cup n\rangle$ are mixed by $a$ for every $m^{\wedge} n \ll c$. We get mixing for all $m^{\wedge} n$ because of our assumption above. Choosing $h \in(c)^{3}$ we obtain that $s^{\wedge}\langle p \cup h(2)\rangle$ and $s^{\wedge}\langle q \cup h(0) \cup h(2)\rangle$ as well as $s^{\wedge}\langle p \cup h(2)\rangle$ and $s^{\wedge}\langle q \cup h(0) \cup h(1) \cup h(2)\rangle$ are mixed by a since $\langle h(0), h(2)\rangle,\langle h(0) \cup h(1), h(2)\rangle \ll c$. Thus, by transitivity of mixing $s^{\wedge}\langle q \cup h(0) \cup h(1) \cup h(2)\rangle$ and $s^{\wedge}\langle q \cup h(0) \cup h(2)\rangle$ are mixed by $a$, contradicting the condition imposed in the lemma. This completes the proof of the claim.

Case (e). $f=\langle 1,1,1\rangle$. To handle case (e) we construct $b_{2} \ll b_{1}$ inductively. To this end we build a sequence $\left\{\left(b_{2}(i), c_{i}\right): i<\omega\right\}$ such that $b_{2} 1(i+1)$ and $c_{i}$ are compatible for every $i<\omega$ with $c_{i} \in \Omega^{\omega}$. Let $b_{2}(0)=b_{1}(0) \cup b_{1}(1)$ and $c_{0}=b_{1} \uparrow 2$. Notice that $b_{2} 11$ and $c_{0}$ are compatible since $f(1)=1$ and $f(2)=1$. Suppose now that $b_{2} 1(k+1)$ and $c_{k}$ have been constructed and are compatible. Since $f(2)=1$, Claim 2.6.2 applies and hence we have that $b_{2} 1(k+1)$ and $c_{k}$ are very compatible. Thus, there exists $\left\langle b_{2}(k+1)\right\rangle \ll c_{k}$ and there exists $c_{k+1} \ll c_{k}$ such that $b_{2} 1(k+2)$ and $c_{k+1}$ are compatible. This completes the construction.

Now we claim that if $m, n \ll b_{2}$ with $m \neq n$ and $\max (m)=\max (n)$, then we have that $s^{\wedge} m$ and $s^{\wedge} n$ are separated by $b_{2}$. To see this, let $b_{2}(k)$ be the last piece of $b_{2}$ occurring in $(m \cup n) \backslash(m \cap n)$. Then we can assume without loss of generality that $m=\left\langle p \cup b_{2}(k) \cup m_{0}\right\rangle$ and $n=\left\langle q \cup m_{0}\right\rangle$ for some $p, q \ll b_{2} 1 k$ and some $m_{0} \ll b_{2}$ with $b_{2}(k)<m_{0}$. Since $b_{2} 1(k+1)$ and $c_{k}$ are compatible, $m_{0} \ll c_{k}$ and $\max \left(p \cup b_{2}(k)\right) \neq \max (q)$ we have that $s^{\wedge}\left\langle p \cup b_{2}(k) \cup m_{0}\right\rangle$ and $s^{\wedge}\left\langle q \cup m_{0}\right\rangle$ are separated by $b_{2}$. Thus, $s^{\wedge} m$ and $s^{\wedge} n$ are separated by $b_{2}$. Since $f(0)=1$ and 
$b_{2} \ll b_{1}$, Claim 2.6.1 applies and we can choose $b \ll b_{2}$ such that $s^{\wedge} m$ and $s^{\wedge} n$ are separated by $b$ whenever $m, n \ll b$ and $\max (m)<\max (n)$.

Finally, notice that if $s^{\wedge} m$ and $s^{\wedge} n$ are separated by $b$, we must have $m \neq n$ by definition of separated. So we can conclude that $s^{\wedge} m$ and $s^{\wedge} n$ are mixed by $b$ iff $m \neq n$.

This completes the proof of case (e) and with it, the proof of Lemma 2.6,

The following definition is based on the five cases of Lemma 2.6

Definition. We say that $s \square$ is strongly mixed by $a$ iff $s \square^{\wedge} m$ and $s \square^{\wedge} n$ are mixed by $a$ for every $m, n \ll a$. Moreover $s$ is min-separated by $a$ iff for every $m, n \ll a$ the sets $s^{\wedge} m$ and $s^{\wedge} n$ are mixed by $a$ iff $\min (m)=\min (n)$. Furthermore, $s \square i s$ max-separated by $a$ iff for every $m, n \ll a$ the sets $s \square^{\wedge} m$ and $s \square^{\wedge} n$ are mixed by $a$ iff $\max (m)=\max (n)$. Moreover we say that $s$ is min-max-separated by $a$ iff for every $m, n \ll a$ the sets $s^{\wedge} m$ and $s^{\wedge} n$ are mixed by $a$ iff $\min (m)=\min (n)$ and $\max (m)=\max (n)$. Finally, $s \square$ is strongly separated by $a$ iff for every $m, n \ll a$ the sets $s \square^{\wedge} m$ and $s \square^{\wedge} n$ are mixed by $a$ iff $m=n$.

Furthermore, we say $s \square$ is separated in some sense by a iff $s \square$ is min-separated, max-separated, min-max-separated or strongly separated by $a$. Moreover $s$ is completely decided by $a$ iff $s$ is strongly mixed by $a$ or $s$ is separated in some sense by $a$.

Corollary 2.7. For every s and a the following properties hold.

(a) Let $s$ be strongly mixed by $a$. Then $s^{\wedge} m \uparrow$ is strongly mixed by a for every $m \ll a$.

(b) Let $s$ be min-separated by $a$. Then $s^{\wedge} m \uparrow$ is strongly mixed by a for every $m \ll a$.

(c) Let $s$ be max-separated by a. Then $s^{\wedge} m \uparrow$ is max-separated by a for every $m \ll a$.

(d) Let $s$ be min-max-separated by $a$. Then $s^{\wedge} m \uparrow$ is max-separated by a for every $m \ll a$.

(e) Let $s$ be strongly separated by $a$. Then $s^{\wedge} m \uparrow$ is strongly separated by a for every $m \ll a$.

Proof. Obvious from the definition.

Lemma 2.8. For every $s$ and $a$ the following properties hold.

(a) Let $s \square$ be strongly mixed by a. Then $s \square$ and $s \square^{\wedge} m \square$ as well as $s \square^{\wedge} m \square$ and $s \square^{\wedge} n \square$ are mixed by a for every $m, n \ll a$.

(b) Let $s$ be min-separated by $a$. Then $s^{\wedge} m \square$ and $s^{\wedge} n \square$ are mixed by a for every $m, n \ll a$ with $\min (m)=\min (n)$.

(c) Let $s \square$ be max-separated by a. Then $s \square$ and $s \square^{\wedge} m \uparrow$ as well as $s \square{ }^{\wedge} m \uparrow$ and $s \square^{\wedge} n \uparrow$ are mixed by a for every $m, n \ll a$.

(d) Let $s$ be min-max-separated by a. Then $s^{\wedge} m \uparrow$ and $s^{\wedge} n \uparrow$ are mixed by a for every $m, n \ll a$ with $\min (m)=\min (n)$.

Proof. Case (a). Let $s \square$ be strongly mixed by $a$. First, we prove that $s \square$ and $s \square^{\wedge} m \square$ are mixed by $a$ for every $m \ll a$. Assume to the contrary that $s \square$ and $s \square^{\wedge} m$, resp. $s \square$ and $s \square^{\wedge} m \uparrow$, are not mixed by $a$ for some $m \ll a$. Hence there exists $b \ll a$ such that $s \square$ and $s \square^{\wedge} m$, resp. $s \square$ and $s \square^{\wedge} m \uparrow$, are separated by $b$. Since $s \square$ is strongly mixed by $a$, by Corollary 2.2 we get that $s \square^{\wedge} m$ and $s \square^{\wedge} n$ are also mixed by $b$ for every $m, n \ll a$. 
Now choose $k$ minimal such that $m<b(k)$. By definition of separation we must have that $s \square^{\wedge}\langle b(k)\rangle$ and $s \square^{\wedge} m$, resp. $s \square^{\wedge}\langle b(k)\rangle$ and $s \square^{\wedge} m \uparrow^{\wedge}\langle b(k)\rangle$, are separated by $b$. However, since $\langle b(k)\rangle \ll a$, both facts contradict that $s \square$ is strongly mixed by $a$.

By transitivity of mixing, the second assertion, that $s \square^{\wedge} m \square$ and $s \square^{\wedge} n \square$ are mixed by $a$ for every $m, n \ll a$, follows from the first one.

Case (b). Let $s$ be min-separated by $a$. Assume to the contrary that $s^{\wedge} m$ and $s^{\wedge} n \uparrow$, resp. $s^{\wedge} m \uparrow$ and $s^{\wedge} n \uparrow$, are not mixed by $a$ for some $m, n \ll a$ with $\min (m)=\min (n)$. Hence there exists $b \ll a$ such that $s^{\wedge} m$ and $s^{\wedge} n \uparrow$, resp. $s^{\wedge} m \uparrow$ and $s^{\wedge} n \uparrow$, are separated by $b$. Since $s$ is min-separated by $a$, by Corollary 2.2 we get that $s^{\wedge} m$ and $s^{\wedge} n$ are also mixed by $b$ for every $m, n \ll a$ with $\min (m)=\min (n)$.

Now choose $k$ minimal such that $m, n<b(k)$. By definition of separation we must have that $s^{\wedge} m$ and $s^{\wedge} n \uparrow^{\wedge}\langle b(k)\rangle$, resp. $s^{\wedge} m \uparrow^{\wedge}\langle b(k)\rangle$ and $s^{\wedge} n \uparrow^{\wedge}\langle b(k)\rangle$, are separated by $b$. However, since $\langle b(k)\rangle \ll a$, both facts contradict that $s$ is min-separated by $a$.

Case (c). Let $s \square$ be max-separated by $a$. First, we prove that $s \square$ and $s \square^{\wedge} m \uparrow$ are mixed by $a$ for every $m \ll a$. Assume to the contrary that $s \square$ and $s \square^{\wedge} m \uparrow$ are not mixed by $a$ for some $m \ll a$. Hence there exists $b \ll a$ such that $s \square$ and $s \square^{\wedge} m \uparrow$ are separated by $b$. Since $s \square$ is max-separated by $a$, by Corollary 2.2 we get that $s \square^{\wedge} m$ and $s \square^{\wedge} n$ are also mixed by $b$ for every $m, n \ll a$ with $\max (m)=\max (n)$.

Now choose $k$ minimal such that $m<b(k)$. By definition of separation we must have that $s \square^{\wedge}\langle b(k)\rangle$ and $s \square^{\wedge} m \uparrow^{\wedge}\langle b(k)\rangle$ are separated by $b$. However, since $\langle b(k)\rangle \ll a$, this contradicts that $s \square$ is max-separated by $a$.

By transitivity of mixing, the second assertion, that $s \square^{\wedge} m \uparrow$ and $s \square^{\wedge} n \uparrow$ are mixed by $a$ for every $m, n \ll a$, follows from the first one.

Case (d). Let $s$ be min-max-separated by $a$. Assume to the contrary that $s^{\wedge} m \uparrow$ and $s^{\wedge} n \uparrow$ are not mixed by $a$ for some $m, n \ll a$ with $\min (m)=\min (n)$. Hence there exists $b \ll a$ such that $s^{\wedge} m \uparrow$ and $s^{\wedge} n \uparrow$ are separated by $b$. Since $s$ is min-max-separated by $a$, by Corollary 2.2 we get that $s^{\wedge} m$ and $s^{\wedge} n$ are also mixed by $b$ for every $m, n \ll a$ with $\min (m)=\min (n)$ and $\max (m)=\max (n)$.

Now choose $k$ minimal such that $m, n<b(k)$. By definition of separation we must have that $s^{\wedge} m \uparrow^{\wedge}\langle b(k)\rangle$ and $s^{\wedge} n \uparrow^{\wedge}\langle b(k)\rangle$ are separated by $b$. However, since $\langle b(k)\rangle \ll a$, this contradicts that $s$ is min-max-separated by $a$.

Lemma 2.9. For every a there exists $b \ll a$ which completely decides every $s \ll b$.

Proof. Inductively, we construct $b_{j} \in \Omega^{\omega}$ for every $j<\omega$. By Lemma 2.6 there exists $b_{0} \ll a$ such that $b_{0}$ completely decides $\varnothing$. Assume by induction that $b_{0}, \ldots, b_{j}$ have been constructed such that for every $i \leq j$ and all $s \ll\left\langle b_{k}(0): k<i\right\rangle$ the set $b_{i}$ completely decides $s$. Some applications of Lemma 2.6 yield $b_{j+1} \ll b_{j} \uparrow 1$ such that the inductive assumption is also satisfied for $b_{0}, \ldots, b_{j+1}$. Then $b=\left\langle b_{j}(0): j \in \omega\right\rangle$ has the desired properties.

Definition. $a$ is canonical for $\Delta$ iff it has the following properties:

(a) The mapping $\Delta 1(a)^{\omega}$ is continuous.

(b) If $s, t \ll a$, then $s \square$ and $t \square$ are decided by $a$.

(c) Every $s \ll a$ is completely decided by $a$.

(d) Let $s, t \ll a$. Then $s \square$ and $s \square^{\wedge} m \square$ are either mixed by $a$ for all $m \ll a$ or separated by $a$ for all $m \ll a$. Equally $s \square^{\wedge} m \square$ and $s \square^{\wedge} m \square, s \square^{\wedge} m \square$ and $t \square^{\wedge} m \square, s \square^{\wedge} m \square$ and $s \square^{\wedge} m \square^{\wedge} n \square$ as well as $s \square^{\wedge} m \square$ and $t \square^{\wedge} m \square^{\wedge} n \square$ 
are in each case either mixed by $a$ for all $m, n \ll a$ or separated by $a$ for all $m, n \ll a$.

(e) If $s \ll a$, then either for every $x \ll a$ and all $k \in \omega$ the set $s \square^{\wedge}(x 1 k)$ is strongly mixed by $a$ or for every $x \ll a$ there exists $k \in \omega$ such that $s \square^{\wedge}(x 1 k)$ is separated in some sense by $a$.

(f) There exists $b$ with $a=\langle b(3 i) \cup b(3 i+1) \cup b(3 i+2): i<\omega\rangle$ such that properties (a) to (e) are even true for $b$ instead of $a$.

Lemma 2.10. There exists a which is canonical for $\Delta$.

Proof. First, observe by Corollary 2.2 that if $s \square$ and $t \square$ are decided by $a$, then they are also decided by each $b \ll a$, and $b$ decides in the same way as $a$ does. Hence by Lemmas 2.1, 2.4 and 2.9 we are guaranteed that there exists $b_{0}$, which satisfies properties (a) to (c) of canonical.

Now we turn to property (d). Inductively, we construct $c_{j} \in \Omega^{\omega}$ for every $j<\omega$. By Lemma 2.5 we can find $c_{0} \ll b_{0}$ such that the sets $\varnothing$ and $\varnothing^{\wedge} m$ are either mixed by $c_{0}$ for every $m \ll c_{0}$ or separated by $c_{0}$ for every $m \ll c_{0}$. Assume that $c_{0}, \ldots, c_{j}$ have been constructed such that for all $i \leq j$ and for all $s \ll\left\langle c_{l}(0): l<i\right\rangle$ the sets $s$ and $s^{\wedge} m$ are either mixed by $c_{i}$ for every $m \ll c_{i}$ or separated by $c_{i}$ for every $m \ll c_{i}$. Again, invoking Lemma 2.5 there exists $c_{j+1} \ll c_{j}\lceil 1$ such that the inductive assumption is also satisfied for $c_{0}, \ldots, c_{j+1}$. Then $b_{1}=\left\langle c_{j}(0): j \in \omega\right\rangle$ has the desired property. Applying some similar inductions, we get $b_{1}$ fulfilling (a) to (d) of canonical.

Now we turn to property (e). Inductively, we construct $c_{j} \in \Omega^{\omega}$ for every $j<\omega$. Consider the set $C=\left\{x \ll b_{1}: \forall k \in \omega x 1 k\right.$ is strongly mixed by $\left.b_{1}\right\}$. Since $C$ is closed, by Theorem 1.15 there exists $c_{0} \ll b_{1}$ such that $\left(c_{0}\right)^{\omega} \subseteq C$ or $\left(c_{0}\right)^{\omega} \subseteq \Omega^{\omega} \backslash C$. Assume that $c_{0}, \ldots, c_{j}$ have been constructed such that for all $i \leq j$ and for all $s \ll\left\langle c_{l}(0): l<i\right\rangle$ either for every $x \ll c_{i}$ and all $k \in \omega$ the set $s \square^{\wedge}(x \mid k)$ is strongly mixed by $c_{i}$ or for every $x \ll c_{i}$ there exists $k \in \omega$ such that $s \square^{\wedge}(x 1 k)$ is separated in some sense by $c_{i}$. For every $s \ll\left\langle c_{l}(0): l<j\right\rangle$ consider the sets $C_{s \square, \square}=\left\{x \ll c_{j}: \forall k \in \omega s \square^{\wedge}\left\langle c_{j}(0)\right\rangle \square^{\wedge}(x 1 k)\right.$ is strongly mixed by $c_{j}$. Again, all $C_{s \square, \square}$ are closed. Hence some applications of Theorem 1.15 yield $c_{j+1} \ll c_{j}\left\lceil 1\right.$ such that the inductive assumption is also satisfied for $c_{0}, \ldots, c_{j+1}$. Then $b=\left\langle c_{j}(0): j \in \omega\right\rangle$ satisfies properties (a) to (e) of canonical.

Finally, let $a=\langle b(3 i) \cup b(3 i+1) \cup b(3 i+2): i<\omega\rangle$. Hence $a$ has properties (a) to (f). This completes the proof.

For the remainder of this paper let $a$ be canonical for $\Delta$.

Lemma 2.11. Let $s \ll a$.

(a) Let $s$ be min-separated by a. If $x, y \ll a$, then $\Delta\left(s^{\wedge} x\right)=\Delta\left(s^{\wedge} y\right)$ implies $\min (x(0))=\min (y(0))$.

(b) Let $s \square$ be max-separated by a. If $x, y \ll a$, then $\Delta\left(s \square^{\wedge} x\right)=\Delta\left(s \square^{\wedge} y\right)$ implies $\max (x(0))=\max (y(0))$.

(c) Let $s$ be min-max-separated by a. If $x, y \ll a$, then $\Delta\left(s^{\wedge} x\right)=\Delta\left(s^{\wedge} y\right)$ implies $\min (x(0))=\min (y(0))$ and $\max (x(0))=\max (y(0))$.

(d) Let $s \square$ be strongly separated by a. If $x, y \ll a$, then $\Delta\left(s \square^{\wedge} x\right)=\Delta\left(s \square^{\wedge} y\right)$ implies that there exists $k$ such that $x(0)=y(0) \cap k$ or $y(0)=x(0) \cap k$, i.e., either $x(0)$ is an initial segment of $y(0)$ or conversely. 
Proof. Let $x, y \ll a$ be such that $\Delta\left(s \square^{\wedge} x\right)=\Delta\left(s \square^{\wedge} y\right)$. Notice that we can assume without loss of generality that $\max (x(0))<\max (y(0))$; since $\max (x(0))=$ $\max (y(0))$ together with the hypothesis of each of the four cases implies that $s \square^{\wedge}\langle x(0)\rangle$ and $s \square^{\wedge}\langle y(0)\rangle$ are mixed by $a$, and the assertion follows by Lemma 2.6

First of all, we show that if $s \square$ is separated in some sense by $a$ and $\max (x(0))<$ $\max (y(0))$, we must have that $\min (x(0))=\min (y(0))$. For that purpose assume to the contrary that $\min (x(0)) \neq \min (y(0))$. We distinguish three cases.

First, let $\max (x(0))<\min (y(0))$. Since $\Delta\left(s \square^{\wedge} x\right)=\Delta\left(s \square^{\wedge} y\right)$, we have that $s \square^{\wedge}\langle x(0)\rangle$ and $s \square$ are mixed by $a$. Hence by (d) of canonical we must have that $s \square^{\wedge} m$ and $s \square$ are mixed by $a$ for all $m \ll a$. By transitivity of mixing it follows that $s \square^{\wedge} m$ and $s \square^{\wedge} n$ are mixed by $a$ for every $m, n \ll a$. But this contradicts that $s \square$ is separated in some sense by $a$.

Next, suppose that $\min (x(0))<\min (y(0))$ and $\max (x(0))>\min (y(0))$. Let $v$ be the part of $x(0)$ below $\min (y(0))$. Since $\Delta\left(s \square^{\wedge} x\right)=\Delta\left(s \square^{\wedge} y\right)$, we have that $s \square \wedge\langle v\rangle \uparrow$ and $s \square$ are mixed by $a$. Thus, by (d) of canonical we must have that $s \square^{\wedge} m \uparrow$ and $s \square$ are mixed by $a$ for all $m \ll a$. Now let $w$ denote the part of $y(0)$ less than or equal to $\max (x(0))$. Hence we have that $s \square^{\wedge}\langle x(0)\rangle$ and $s \square^{\wedge}\langle w\rangle \uparrow$ are mixed by $a$. By transitivity of mixing $s \square^{\wedge}\langle x(0)\rangle$ and $s \square$ are mixed by $a$. Therefore, by (d) of canonical we must have that $s \square^{\wedge} m$ and $s \square$ are mixed by $a$ for all $m \ll a$. Again, by transitivity of mixing it follows that $s \square^{\wedge} m$ and $s \square^{\wedge} n$ are mixed by $a$ for every $m, n \ll a$. But this contradicts that $s \square$ is separated in some sense by $a$.

Finally, assume that $\min (x(0))>\min (y(0))$. Let $v$ be the part of $y(0)$ below $\min (x(0))$. Since $\Delta\left(s \square^{\wedge} x\right)=\Delta\left(s \square^{\wedge} y\right)$, we have that $s \square$ and $s \square^{\wedge}\langle v\rangle \uparrow$ are mixed by $a$. Thus, by (d) of canonical we must have that $s \square$ and $s \square^{\wedge} m \uparrow$ are mixed by $a$ for all $m \ll a$. Now let $w$ denote the part of $y(0)$ less than or equal to $\max (x(0))$. Hence we have that $s \square^{\wedge}\langle x(0)\rangle$ and $s \square^{\wedge}\langle w\rangle \uparrow$ are mixed by $a$. By transitivity of mixing $s \square^{\wedge}\langle x(0)\rangle$ and $s \square$ are mixed by $a$. Therefore, by (d) of canonical we must have that $s \square^{\wedge} m$ and $s \square$ are mixed by $a$ for all $m \ll a$. Again, by transitivity of mixing it follows that $s \square^{\wedge} m$ and $s \square^{\wedge} n$ are mixed by $a$ for every $m, n \ll a$. But this contradicts that $s \square$ is separated in some sense by $a$.

Therewith we must have $\min (x(0))=\min (y(0))$. This already proves case (a) of this lemma.

Now we prove cases (b) and (c) in one step. For that purpose let $s \square$ be maxseparated or min-max-separated by $a$. Recall that we can assume without loss of generality that $\min (x(0))=\min (y(0))$ and $\max (x(0))<\max (y(0))$.

Let $v$ be the part of $y(0)$ less than or equal to $\max (x(0))$. Hence we have that $s \square^{\wedge}\langle x(0)\rangle$ and $s \square^{\wedge}\langle v\rangle \uparrow$ are mixed by $a$. Additionally, cases (c) and (d) of Lemma 2.8 yield that $s \square^{\wedge} m \uparrow$ and $s \square^{\wedge} n \uparrow$ are mixed by $a$ for all $m, n \ll a$ with $\min (m)=\min (n)$. Therefore $s \square^{\wedge}\langle v\rangle \uparrow$ and $s \square^{\wedge}\langle x(0)\rangle \uparrow$ are mixed by $a$, because $\min (v)=\min (x(0))$. By transitivity of mixing we get that $s \square^{\wedge}\langle x(0)\rangle$ and $s \square^{\wedge}\langle x(0)\rangle \uparrow$ are mixed by $a$. Moreover by (d) of canonical we must have that $s \square^{\wedge} m$ and $s \square^{\wedge} m \uparrow$ are mixed by $a$ for every $m \ll a$. Altogether, we have that $s \square^{\wedge}\langle x(0)\rangle$ and $s \square^{\wedge}\langle x(0)\rangle \uparrow, s \square^{\wedge}\langle x(0)\rangle \uparrow$ and $s \square^{\wedge}\langle y(0)\rangle \uparrow$ as well as $s \square^{\wedge}\langle y(0)\rangle \uparrow$ and $s \square^{\wedge}\langle y(0)\rangle$ are mixed by $a$. Again, by transitivity of mixing we obtain that $s \square^{\wedge}\langle x(0)\rangle$ and $s \square^{\wedge}\langle y(0)\rangle$ are mixed by $a$. But this contradicts the fact that $s \square$ is max-separated or min-max-separated by $a$.

Hence we must have that $\max (x(0))=\max (y(0))$, and the assertion follows by Lemma 2.6 
Finally, we prove case (d) of this lemma. For that purpose let $s \square$ be strongly separated by $a$. Recall that we can assume without loss of generality that $\min (x(0))=$ $\min (y(0))$ and $\max (x(0))<\max (y(0))$. Suppose to the contrary that $x(0)$ is not an initial segment of $y(0)$.

Let $b$ with $a \ll b$ be as in (f) of canonical. Moreover let $v$ denote the longest common initial segment of $x(0)$ and $y(0)$. Choose $k$ with $\min (x(0) \triangle y(0)) \in b(k)$. Since $x(0)$ is not an initial segment of $y(0)$, we have that $s \square^{\wedge}\langle v\rangle \uparrow$ and $s \square^{\wedge}\langle v\rangle \uparrow$ ${ }^{\wedge}\langle b(k)\rangle \uparrow$ are mixed by $b$. Hence by (d) of canonical we must have that $s \square^{\wedge} m \uparrow$ and $s \square^{\wedge} m \uparrow^{\wedge} n \uparrow$ are mixed by $b$ for all $m, n \ll b$. Furthermore, let $w$ denote the part of $y(0)$ less than or equal to $\max (x(0))$. Therewith we get that $s \square^{\wedge}\langle x(0)\rangle$ and $s \square^{\wedge}\langle w\rangle \uparrow$ are mixed by $b$, too. Since $v$ is an initial segment of $w$, we get with the result above that $s \square^{\wedge}\langle w\rangle \uparrow$ and $s \square^{\wedge}\langle v\rangle \uparrow$ are mixed by $b$. Hence by transitivity of mixing $s \square^{\wedge}\langle x(0)\rangle$ and $s \square^{\wedge}\langle v\rangle \uparrow$ are mixed by $b$. Moreover since $v$ is an initial segment of $x(0)$, property (d) of canonical yields that $s \square^{\wedge} m \uparrow$ and $s \square^{\wedge} m \uparrow^{\wedge} n$ are mixed by $b$ for all $m, n \ll b$. Thus, equally $s \square^{\wedge}\langle y(0)\rangle$ and $s \square^{\wedge}\langle v\rangle \uparrow$ are mixed by $b$, and by transitivity we obtain that $s \square^{\wedge}\langle x(0)\rangle$ and $s \square^{\wedge}\langle y(0)\rangle$ are mixed by $b$. Since $a \ll b$, we must have that $s \square^{\wedge}\langle x(0)\rangle$ and $s \square^{\wedge}\langle y(0)\rangle$ are also mixed by $a$. But this contradicts that $s \square$ is strongly separated by $a$.

Hence we must have that $x(0)$ is an initial segment of $y(0)$. This completes the proof.

Lemma 2.12. Let $s, t \ll a$. Suppose $s \square$ and $t \square$ are mixed by $a$ and $s \square$ is separated in some sense by $a$. If $x, y \ll a$ such that $\Delta\left(s \square^{\wedge} x\right)=\Delta\left(t \square^{\wedge} y\right)$, then $\max (x(0))>$ $\min (y(0))$.

Proof. Let $x, y \ll a$ be such that $\Delta\left(s \square^{\wedge} x\right)=\Delta\left(t \square^{\wedge} y\right)$. Assume to the contrary that $\max (x(0))<\min (y(0))$. Note that $\max (x(0))=\min (y(0))$ is impossible by (f) of canonical.

Choose $0<k \leq d o m(t)$ maximal with $\max (t(k-1)) \leq \max (x(0))$ if possible, otherwise choose $k=0$. Moreover if $k<\operatorname{dom}(t)$, let $v$ denote the part of $t(k)$ less than or equal to $\max (x(0))$. Thus, if $k=\operatorname{dom}(t)$ or $v=\varnothing$, we have that $s \square^{\wedge}\langle x(0)\rangle$ and $t 1 k \square$ are mixed by $a$. Otherwise we have that $s \square^{\wedge}\langle x(0)\rangle$ and $t 1 k^{\wedge}\langle v\rangle \uparrow$ are mixed by $a$.

Moreover since $s \square$ and $t \square$ are mixed by $a$, there exist $x_{0}, y_{0} \ll a$ with $s<x_{0}, y_{0}$ and $t<x_{0}, y_{0}$ such that $\Delta\left(s \square^{\wedge} x_{0}\right)=\Delta\left(t \square^{\wedge} y_{0}\right)$.

Now assume that we are in the first case, where $s \square^{\wedge}\langle x(0)\rangle$ and $t 1 k \square$ are mixed by $a$. If $k<\operatorname{dom}(t)$, we can choose $y_{1} \ll a$ by $y_{1}=\langle t(i): k \leq i<\operatorname{dom}(t)\rangle^{\wedge} y_{0}$ such that $\Delta\left(s \square^{\wedge} x_{0}\right)=\Delta\left(t 1 k \square^{\wedge} y_{1}\right)$. By choice of $k$ we have $s<x_{0}, y_{1}$ and $t 1 k<x_{0}, y_{1}$. Hence by (b) of canonical we must have that $s \square$ and $t 1 k \square$ are mixed by $a$.

Next, suppose that we are in the case where $s \square^{\wedge}\langle x(0)\rangle$ and $t 1 k^{\wedge}\langle v\rangle \uparrow$ are mixed by $a$. Let $w$ be the part of $t(k)$ above $\max (v)$. If $k<\operatorname{dom}(t)-1$, choose $y_{1} \ll a$ by $y_{1}=\langle w\rangle^{\wedge}\langle t(i): k<i<\operatorname{dom}(t)\rangle^{\wedge} y_{0}$, otherwise choose $y_{1}=\langle w\rangle^{\wedge} y_{0}$. Therewith we have that $\Delta\left(s \square^{\wedge} x_{0}\right)=\Delta\left(t 1 k^{\wedge}\langle v\rangle \uparrow^{\wedge} y_{1}\right)$ with $s<x_{0}, y_{1}$ and $t 1 k^{\wedge}\langle v\rangle<x_{0}, y_{1}$. Thus, by (b) of canonical we get that $s \square$ and $t 1 k^{\wedge}\langle v\rangle \uparrow$ are mixed by $a$.

Since $s \square$ and $t \square$ are mixed by $a$, by transitivity of mixing we can conclude that $s \square^{\wedge}\langle x(0)\rangle$ and $s \square$ are mixed by $a$, contradicting all cases of Lemma 2.11.

Lemma 2.13. Let $s, t \ll a$. If $x, y \ll a$ with $\min (x(0))=\min (y(0))$ such that $\Delta\left(s \square{ }^{\wedge} x\right)=\Delta\left(t \square^{\wedge} y\right)$, then $s \square^{\wedge} m \uparrow$ and $t \square^{\wedge} m \uparrow$ are mixed by a for every $m \ll a$. 
Proof. Let $b$ with $a \ll b$ be as in (f) of canonical. Choose $k$ with $\min (x(0)) \in b(k)$. Since $\Delta\left(s \square^{\wedge} x\right)=\Delta\left(t \square^{\wedge} y\right)$, we have that $s \square^{\wedge}\langle b(k)\rangle \uparrow$ and $t \square^{\wedge}\langle b(k)\rangle \uparrow$ are mixed by $b$. By (d) of canonical we must have that $s \square^{\wedge} m \uparrow$ and $t \square^{\wedge} m \uparrow$ are mixed by $b$ for every $m \ll b$. Since $a \ll b$, by Corollary 2.2 we also have that $s \square^{\wedge} m \uparrow$ and $t \square^{\wedge} m \uparrow$ are mixed by $a$ for every $m \ll a$.

Lemma 2.14. Let $s, t \ll a$. Suppose $s$ and $t$ are mixed by $a$ and both $s$ and $t$ are min-separated by $a$. If $x, y \ll a$ such that $\Delta\left(s^{\wedge} x\right)=\Delta\left(t^{\wedge} y\right)$, then $\min (x(0))=$ $\min (y(0))$.

Proof. Let $x, y \ll a$ be such that $\Delta\left(s^{\wedge} x\right)=\Delta\left(t^{\wedge} y\right)$. Assume to the contrary that $\min (x(0)) \neq \min (y(0))$. By symmetry we can suppose without loss of generality that $\min (x(0))<\min (y(0))$. Moreover by Lemma 2.12 it suffices to prove that the assumption that $\min (x(0))<\min (y(0))$ and $\max (x(0))>\min (y(0))$ leads to a contradiction.

Let $v$ be the part of $x(0)$ below $\min (y(0))$. Since $\Delta\left(s^{\wedge} x\right)=\Delta\left(t^{\wedge} y\right)$, we must have that $s^{\wedge}\langle v\rangle \uparrow$ and $t$ are mixed by $a$. Moreover since $s$ and $t$ are mixed by $a$, by transitivity of mixing we get that $s^{\wedge}\langle v\rangle \uparrow$ and $s$ are mixed by $a$, contradicting case (a) of Lemma 2.11.

Lemma 2.15. Let $s, t \ll a$. Suppose $s$ and $t$ are mixed by $a$ and both $s$ and $t$ are min-separated by $a$. Then $s^{\wedge} m$ and $t^{\wedge} n$ are mixed by a for all $m, n \ll a$ with $\min (m)=\min (n)$.

Proof. Since $s$ and $t$ are mixed by $a$, there exist $x, y \ll a$ such that $\Delta\left(s^{\wedge} x\right)=\Delta\left(t^{\wedge} y\right)$. By Lemma 2.14 we have that $\min (x(0))=\min (y(0))$.

Moreover by Lemma 2.13 we get that $s^{\wedge} m \uparrow$ and $t^{\wedge} m \uparrow$ are mixed by $a$ for every $m \ll a$. Additionally, case (b) of Lemma 2.8 yields that $s^{\wedge} m$ and $s^{\wedge} m \uparrow$ as well as $t^{\wedge} m \uparrow$ and $t^{\wedge} n$ are mixed by $a$ for all $m, n \ll a$ with $\min (m)=\min (n)$. Thus, by transitivity of mixing we get that $s^{\wedge} m$ and $t^{\wedge} n$ are mixed by $a$ for every $m, n \ll a$ with $\min (m)=\min (n)$.

Lemma 2.16. Let $s, t \ll a$. Suppose $s \square$ and $t \square$ are mixed by $a$ and both $s \square$ and $t \square$ are max-separated by $a$. If $x, y \ll a$ such that $\Delta\left(s \square^{\wedge} x\right)=\Delta\left(t \square^{\wedge} y\right)$, then $\max (x(0))=\max (y(0))$.

Proof. Let $x, y \ll a$ be such that $\Delta\left(s \square^{\wedge} x\right)=\Delta\left(t \square^{\wedge} y\right)$. Assume to the contrary that $\max (x(0)) \neq \max (y(0))$. By symmetry we can suppose without loss of generality that $\max (x(0))<\max (y(0))$. Moreover by Lemma 2.12 it suffices to prove that the assumption that $\max (x(0))<\max (y(0))$ and $\max (x(0))>\min (y(0))$ leads to a contradiction.

So let $w$ be the part of $y(0)$ less than or equal to $\max (x(0))$. Since $\Delta\left(s \square^{\wedge} x\right)=$ $\Delta\left(t \square^{\wedge} y\right)$, we have that $s \square^{\wedge}\langle x(0)\rangle$ and $t \square^{\wedge}\langle w\rangle \uparrow$ are mixed by $a$. Additionally, case (c) of Lemma 2.8 yields that $t \square$ and $t \square^{\wedge}\langle w\rangle \uparrow$ are mixed by $a$. Moreover since $s \square$ and $t \square$ are mixed by $a$, by transitivity of mixing it follows that $s \square$ and $s \square^{\wedge}\langle x(0)\rangle$ are mixed by $a$, contradicting case (b) of Lemma 2.11

Lemma 2.17. Let $s, t \ll a$. Suppose $s \square$ and $t \square$ are mixed by $a$ and both $s \square$ and $t \square$ are max-separated by $a$. Then $s \square^{\wedge} m$ and $t \square^{\wedge} n$ are mixed by a for all $m, n \ll a$ with $\max (m)=\max (n)$.

Proof. Since $s \square$ and $t \square$ are mixed by $a$, there exist $x, y \ll a$ such that $\Delta\left(s \square^{\wedge} x\right)=$ $\Delta\left(t \square^{\wedge} y\right)$. By Lemma 2.16 we have that $\max (x(0))=\max (y(0))$. Hence by definition 
of mixing we must have that $s \square^{\wedge}\langle x(0)\rangle$ and $t \square^{\wedge}\langle y(0)\rangle$ are mixed by $a$. Moreover we have that $t \square^{\wedge}\langle x(0)\rangle$ and $t \square^{\wedge}\langle y(0)\rangle$ are mixed by $a$, because $t \square$ is max-separated by $a$. By transitivity of mixing we get that $s \square^{\wedge}\langle x(0)\rangle$ and $t \square^{\wedge}\langle x(0)\rangle$ are mixed by $a$. Thus, (d) of canonical yields that $s \square^{\wedge} m$ and $t \square^{\wedge} m$ are mixed by $a$ for all $m \ll a$.

Again, since $t \square$ is max-separated by $a$, we have that $t \square^{\wedge} m$ and $t \square^{\wedge} n$ are mixed by $a$ for every $m, n \ll a$ with $\max (m)=\max (n)$. Finally, by transitivity of mixing we obtain that $s \square^{\wedge} m$ and $t \square^{\wedge} n$ are mixed by $a$ for all $m, n \ll a$ with $\max (m)=\max (n)$.

Lemma 2.18. Let $s, t \ll a$. Suppose $s$ and $t$ are mixed by $a$ and both $s$ and $t$ are min-max-separated by $a$. If $x, y \ll a$ such that $\Delta\left(s^{\wedge} x\right)=\Delta\left(t^{\wedge} y\right)$, then $\min (x(0))=\min (y(0))$ and $\max (x(0))=\max (y(0))$.

Proof. Let $x, y \ll a$ be such that $\Delta\left(s^{\wedge} x\right)=\Delta\left(t^{\wedge} y\right)$. First, assume to the contrary that $\min (x(0)) \neq \min (y(0))$. By symmetry we can suppose without loss of generality that $\min (x(0))<\min (y(0))$. Moreover by Lemma 2.12 it suffices to prove that the assumption that $\min (x(0))<\min (y(0))$ and $\max (x(0))>\min (y(0))$ leads to a contradiction.

Let $v$ be the part of $x(0)$ below $\min (y(0))$. Since $\Delta\left(s^{\wedge} x\right)=\Delta\left(t^{\wedge} y\right)$ we must have that $s^{\wedge}\langle v\rangle \uparrow$ and $t$ are mixed by $a$. Since $s$ and $t$ are mixed by $a$, by transitivity of mixing we get that $s^{\wedge}\langle v\rangle \uparrow$ and $s$ are mixed by $a$, contradicting case (c) of Lemma 2.11.

Hence we must have $\min (x(0))=\min (y(0))$. Now assume to the contrary that $\max (x(0)) \neq \max (y(0))$. Equally by symmetry we can suppose without loss of generality that $\max (x(0))<\max (y(0))$.

Let $w$ be the part of $y(0)$ less than or equal to $\max (x(0))$. Therewith we have that $s^{\wedge}\langle x(0)\rangle$ and $t^{\wedge}\langle w\rangle \uparrow$ are mixed by $a$. Since $\min (x(0))=\min (y(0))$, Lemma 2.13 yields that $s^{\wedge}\langle w\rangle \uparrow$ and $t^{\wedge}\langle w\rangle \uparrow$ are mixed by $a$. By transitivity of mixing we get that $s^{\wedge}\langle x(0)\rangle$ and $s^{\wedge}\langle w\rangle \uparrow$ are mixed by $a$, contradicting case (c) of Lemma 2.11 This completes the proof of the lemma.

Lemma 2.19. Let $s, t \ll a$. Suppose $s$ and $t$ are mixed by $a$ and both $s$ and $t$ are min-max-separated by $a$. Then $s^{\wedge} m \uparrow$ and $t^{\wedge} m \uparrow$ are mixed by a for every $m \ll a$. Moreover $s^{\wedge} m$ and $t^{\wedge} n$ are mixed by a for all $m, n \ll a$ with $\min (m)=\min (n)$ and $\max (m)=\max (n)$.

Proof. Since $s$ and $t$ are mixed by $a$, there exist $x, y \ll a$ such that $\Delta\left(s^{\wedge} x\right)=\Delta\left(t^{\wedge} y\right)$. By Lemma 2.18 we get that $\min (x(0))=\min (y(0))$ and $\max (x(0))=\max (y(0))$. Therefore, Lemma 2.13 yields that $s^{\wedge} m \uparrow$ and $t^{\wedge} m \uparrow$ are mixed by $a$ for every $m \ll a$, which is our first assertion.

Additionally, by definition of mixing we have that $s^{\wedge}\langle x(0)\rangle$ and $t^{\wedge}\langle y(0)\rangle$ are mixed by $a$. Moreover we have that $t^{\wedge}\langle x(0)\rangle$ and $t^{\wedge}\langle y(0)\rangle$ are mixed by $a$, because $t$ is min-max-separated by $a$. By transitivity of mixing we get that $s^{\wedge}\langle x(0)\rangle$ and $t^{\wedge}\langle x(0)\rangle$ are mixed by $a$. Thus, (d) of canonical yields that $s^{\wedge} m$ and $t^{\wedge} m$ are mixed by $a$ for all $m \ll a$.

Again, since $t$ is min-max-separated by $a$, we have that $t^{\wedge} m$ and $t^{\wedge} n$ are mixed by $a$ for every $m, n \ll a$ with $\min (m)=\min (n)$ and $\max (m)=\max (n)$. Finally, by transitivity of mixing we obtain that $s^{\wedge} m$ and $t^{\wedge} n$ are mixed by $a$ for all $m, n \ll a$ with $\min (m)=\min (n)$ and $\max (m)=\max (n)$. 
Lemma 2.20. Let $s, t \ll a$. Suppose $s \square$ and $t \square$ are mixed by a and both $s \square$ and $t \square$ are strongly separated by $a$. If $x, y \ll a$ such that $\Delta\left(s \square^{\wedge} x\right)=\Delta\left(t \square^{\wedge} y\right)$, then there exists $k$ such that $x(0)=y(0) \cap k$ or $y(0)=x(0) \cap k$, i.e., either $x(0)$ is an initial segment of $y(0)$ or conversely.

Proof. Let $x, y \ll a$ be such that $\Delta\left(s \square^{\wedge} x\right)=\Delta\left(t \square^{\wedge} y\right)$. First, assume to the contrary that $\min (x(0)) \neq \min (y(0))$. By symmetry we can suppose without loss of generality that $\min (x(0))<\min (y(0))$. Moreover by Lemma 2.12 it suffices to prove that the assumption that $\min (x(0))<\min (y(0))$ and $\max (x(0))>\min (y(0))$ leads to a contradiction.

Let $v$ be the part of $x(0)$ below $\min (y(0))$. Since $\Delta\left(s \square^{\wedge} x\right)=\Delta\left(t \square^{\wedge} y\right)$, we must have that $s \square^{\wedge}\langle v\rangle \uparrow$ and $t \square$ are mixed by $a$. Moreover since $s \square$ and $t \square$ are mixed by $a$, by transitivity of mixing we get that $s \square^{\wedge}\langle v\rangle \uparrow$ and $s \square$ are mixed by $a$, contradicting case (d) of Lemma 2.11.

Hence we must have that $\min (x(0))=\min (y(0))$. Now assume to the contrary that neither $x(0)$ is an initial segment of $y(0)$ nor conversely. By symmetry we can suppose without loss of generality that $x(0)$ is not an initial segment of $y(0)$.

Let $v$ denote the longest common initial segment of $x(0)$ and $y(0)$. Moreover choose $k$ with $\min (x(0) \Delta y(0)) \in a(k)$. Since $\Delta\left(s \square^{\wedge} x\right)=\Delta\left(t \square^{\wedge} y\right)$, we have that either $s \square^{\wedge}\langle v\rangle \uparrow^{\wedge}\langle a(k)\rangle \square$ and $t \square^{\wedge}\langle v\rangle \uparrow$ or $s \square^{\wedge}\langle v\rangle \uparrow$ and $t \square^{\wedge}\langle v\rangle \uparrow^{\wedge}\langle a(k)\rangle \uparrow$ are mixed by $a$. Additionally, by Lemma 2.13 we have that $s \square^{\wedge}\langle v\rangle \uparrow$ and $t \square^{\wedge}\langle v\rangle \uparrow$ are mixed by $a$, because $\min (x(0))=\min (y(0))$. Thus, by transitivity of mixing we get in the first case that $s \square^{\wedge}\langle v\rangle \uparrow^{\wedge}\langle a(k)\rangle \square$ and $s \square^{\wedge}\langle v\rangle \uparrow$, in the second case that $t \square^{\wedge}\langle v\rangle \uparrow$ and $t \square^{\wedge}\langle v\rangle \uparrow^{\wedge}\langle a(k)\rangle \uparrow$ are mixed by $a$. Both cases contradict case (d) of Lemma 2.11

Now we want to analyse the case that $s \square$ is strongly separated by $a$. Since $a$ is canonical for $\Delta$, we are able to distinguish exactly two possibilities.

Definition. Let $s \ll a$. Suppose that $s \square$ is strongly separated by $a$. We say that $s \square$ is still strongly separated by $a$ iff $s \square^{\wedge} m$ and $s \square^{\wedge} m \uparrow$ are mixed by $a$ for every $m \ll a$. Moreover $s \square$ is very strongly separated by $a$ iff $s \square^{\wedge} m$ and $s \square^{\wedge} m \uparrow$ are separated by $a$ for every $m \ll a$.

Corollary 2.21. Let $s \ll a$.

(a) Let $s \square$ be still strongly separated by a. Then $s \square^{\wedge} m \uparrow$ is still strongly separated by a for every $m \ll a$.

(b) Let $s \square$ be very strongly separated by a. Then $s \square^{\wedge} m \uparrow$ is very strongly separated by a for every $m \ll a$.

Proof. Obvious from the definition.

Lemma 2.22. Let $s, t \ll a$. Suppose $s \square$ and $t \square$ are mixed by $a$ and both $s \square$ and $t \square$ are very strongly separated by $a$. If $x, y \ll a$ such that $\Delta\left(s \square^{\wedge} x\right)=\Delta\left(t \square^{\wedge} y\right)$, then $x(0)=y(0)$.

Proof. Let $x, y \ll a$ be such that $\Delta\left(s \square^{\wedge} x\right)=\Delta\left(t \square^{\wedge} y\right)$. By Lemma 2.20 we have that $x(0)$ is an initial segment of $y(0)$ or conversely. Moreover by symmetry we can suppose without loss of generality that $x(0)$ is an initial segment of $y(0)$.

Assume to the contrary that $\max (x(0))<\max (y(0))$. Since $\Delta\left(s \square^{\wedge} x\right)=\Delta\left(t \square^{\wedge} y\right)$, we have that $s \square^{\wedge}\langle x(0)\rangle$ and $t \square^{\wedge}\langle x(0)\rangle \uparrow$ are mixed by $a$. By (d) of canonical we get that $s \square^{\wedge} m$ and $t \square^{\wedge} m \uparrow$ are mixed by $a$ for all $m \ll a$. Since $\min (x(0))=\min (y(0))$, 
by Lemma 2.13 we also have that $s \square^{\wedge} m \uparrow$ and $t \square^{\wedge} m \uparrow$ are mixed by $a$ for all $m \ll a$. Finally, by transitivity of mixing we can conclude that $s \square^{\wedge} m$ and $s \square^{\wedge} m \uparrow$ are mixed by $a$ for every $m \ll a$. But this contradicts our assumption that $s \square$ is very strongly separated by $a$.

Lemma 2.23. Let $s, t \ll a$. Suppose $s \square$ and $t \square$ are mixed by a and both $s \square$ and $t \square$ are very strongly separated by $a$. Then $s \square^{\wedge} m$ and $t \square^{\wedge} m$ are mixed by a for all $m \ll a$.

Proof. Otherwise by (d) of canonical we would have that $s \square^{\wedge} m$ and $t \square^{\wedge} m$ are separated by $a$ for every $m \ll a$. By Lemma 2.22 this would contradict that $s \square$ and $t \square$ are mixed by $a$, so the assertion follows.

Lemma 2.24. Let $s, t \ll a$. Suppose $s \square$ and $t \square$ are mixed by a and $s \square$ is strongly mixed by a. Moreover assume that $t \square$ is either min-separated, min-max-separated or strongly separated by $a$. If $x, y \ll$ a such that $\Delta\left(s \square^{\wedge} x\right)=\Delta\left(t \square^{\wedge} y\right)$, then $\max (x(0))<\min (y(0))$.

Proof. Let $x, y \ll a$ be such that $\Delta\left(s \square^{\wedge} x\right)=\Delta\left(t \square^{\wedge} y\right)$. Assume to the contrary that $\max (x(0))>\min (y(0))$. By Lemma 2.12 we must have that $\min (x(0))<\max (y(0))$. We distinguish three cases.

For the first case suppose that $\min (x(0))>\min (y(0))$ and $\min (x(0))<\max (y(0))$. Let $v$ denote the part of $y(0)$ below $\min (x(0))$. Since $\Delta\left(s \square^{\wedge} x\right)=\Delta\left(t \square^{\wedge} y\right)$, we have that $s \square$ and $t \square^{\wedge}\langle v\rangle \uparrow$ are mixed by $a$. Moreover since $s \square$ and $t \square$ are mixed by $a$, by transitivity of mixing we get that $t \square$ and $t \square^{\wedge}\langle v\rangle \uparrow$ are mixed by $a$. But this contradicts cases (a), (c) and (d) of Lemma 2.11.

Next, assume that $\min (x(0)) \leq \min (y(0))$ and $\max (x(0)) \geq \max (y(0))$. Let $v$ be the part of $x(0)$ less than or equal to $\max (y(0))$. Since $\Delta\left(s \square^{\wedge} x\right)=\Delta\left(t \square^{\wedge} y\right)$, we have that $s \square^{\wedge}\langle v\rangle \square$ and $t \square^{\wedge}\langle y(0)\rangle$ are mixed by $a$. Additionally, by (a) of Lemma 2.8 we have that $s \square$ and $s \square^{\wedge}\langle v\rangle \square$ are mixed by $a$, because $s \square$ is strongly mixed by $a$. Moreover since $s \square$ and $t \square$ are mixed by $a$, by transitivity of mixing we obtain that $t \square$ and $t \square^{\wedge}\langle y(0)\rangle$ are mixed by $a$. Equally, this contradicts cases (a), (c) and (d) of Lemma 2.11

Finally, suppose that $\min (x(0)) \leq \min (y(0))$ and $\max (x(0))<\max (y(0))$. Let $v$ denote the part of $y(0)$ less than or equal to $\max (x(0))$. Since $\Delta\left(s \square^{\wedge} x\right)=\Delta\left(t \square^{\wedge} y\right)$, we have that $s \square^{\wedge}\langle x(0)\rangle$ and $t \square^{\wedge}\langle v\rangle \uparrow$ are mixed by $a$. Additionally, by (a) of Lemma 2.8 we have that $s \square$ and $s \square^{\wedge}\langle x(0)\rangle$ are mixed by $a$, because $s \square$ is strongly mixed by $a$. Moreover since $s \square$ and $t \square$ are mixed by $a$, by transitivity of mixing we get that $t \square$ and $t \square^{\wedge}\langle v\rangle \uparrow$ are mixed by $a$, a contradiction as above.

Lemma 2.25. Let $s, t \ll a$. Suppose $s \square$ and $t \square$ are mixed by $a, s \square$ is strongly mixed by $a$ and $t \square$ is max-separated by $a$. If $x, y \ll a$ such that $\Delta\left(s \square{ }^{\wedge} x\right)=$ $\Delta\left(t \square^{\wedge} y\right)$, then $\max (x(0))<\max (y(0))$.

Proof. Let $x, y \ll a$ be such that $\Delta\left(s \square^{\wedge} x\right)=\Delta\left(t \square^{\wedge} y\right)$. Assume to the contrary that $\max (x(0)) \geq \max (y(0))$. By Lemma 2.12 we must have $\min (x(0))<\max (y(0))$. We distinguish two cases.

First, suppose that $\max (x(0))>\max (y(0))$ and $\min (x(0))<\max (y(0))$. Let $v$ denote the part of $x(0)$ less than or equal to $\max (y(0))$. Since $\Delta\left(s \square^{\wedge} x\right)=\Delta\left(t \square^{\wedge} y\right)$, we have that $s \square^{\wedge}\langle v\rangle \uparrow$ and $t \square^{\wedge}\langle y(0)\rangle$ are mixed by $a$. Moreover by (a) of Lemma 2.8 we have that $s \square^{\wedge}\langle v\rangle \uparrow$ and $s \square$ are mixed by $a$, because $s \square$ is strongly mixed by $a$. 
Finally, since $s \square$ and $t \square$ are mixed by $a$, by transitivity of mixing we can conclude that $t \square^{\wedge}\langle y(0)\rangle$ and $t \square$ are mixed by $a$. But this contradicts case (b) of Lemma 2.11.

Next, assume that $\max (x(0))=\max (y(0))$. By definition of mixing we have that $s \square^{\wedge}\langle x(0)\rangle$ and $t \square^{\wedge}\langle y(0)\rangle$ are mixed by $a$. Since $s \square$ is strongly mixed by $a$, by (a) of Lemma 2.8 we get that also $s \square^{\wedge}\langle x(0)\rangle$ and $s \square$ are mixed by $a$. Moreover we have that $s \square$ and $t \square$ are mixed by $a$. Therefore, by transitivity of mixing we get that $t \square^{\wedge}\langle y(0)\rangle$ and $t \square$ are mixed by $a$, which equally contradicts case (b) of Lemma 2.11

Lemma 2.26. Let $s, t \ll a$. Suppose $s$ and $t$ are mixed by $a, s$ is min-separated by $a$ and $t$ is min-max-separated by $a$. If $x, y \ll a$ such that $\Delta\left(s^{\wedge} x\right)=\Delta\left(t^{\wedge} y\right)$, then $\min (x(0))=\min (y(0))$ and $\max (x(0))<\max (y(0))$.

Proof. Let $x, y \ll a$ be such that $\Delta\left(s^{\wedge} x\right)=\Delta\left(t^{\wedge} y\right)$. First of all, we prove that we must have $\min (x(0))=\min (y(0))$. For that purpose assume to the contrary that $\min (x(0)) \neq \min (y(0))$. Two applications of Lemma 2.12 yield that $\max (x(0))>$ $\min (y(0))$ and $\min (x(0))<\max (y(0))$. We distinguish two more cases.

First, suppose that $\min (x(0))<\min (y(0))$ and $\max (x(0))>\min (y(0))$. Let $v$ denote the part of $x(0)$ below $\min (y(0))$. Since $\Delta\left(s^{\wedge} x\right)=\Delta\left(t^{\wedge} y\right)$, we have that $s^{\wedge}\langle v\rangle \uparrow$ and $t$ are mixed by $a$. Moreover $s$ and $t$ are mixed by $a$. Hence by transitivity of mixing we get that $s^{\wedge}\langle v\rangle \uparrow$ and $s$ are mixed by $a$. But this contradicts case (a) of Lemma 2.11.

Next, assume that $\min (x(0))>\min (y(0))$ and $\min (x(0))<\max (y(0))$. Let $v$ be the part of $y(0)$ below $\min (x(0))$. Since $\Delta\left(s^{\wedge} x\right)=\Delta\left(t^{\wedge} y\right)$, we have that $s$ and $t^{\wedge}\langle v\rangle \uparrow$ are mixed by $a$. Moreover $s$ and $t$ are mixed by $a$. Hence by transitivity of mixing we get that $t$ and $t^{\wedge}\langle v\rangle \uparrow$ are mixed by $a$. This contradicts case (c) of Lemma 2.11

Hence we have $\min (x(0))=\min (y(0))$. Now we show that we also have that $\max (x(0))<\max (y(0))$. For that purpose assume to the contrary that $\max (x(0)) \geq$ $\max (y(0))$.

Let $v$ denote the part of $x(0)$ less than or equal to $\max (y(0))$. Since $\Delta\left(s^{\wedge} x\right)=$ $\Delta\left(t^{\wedge} y\right)$, we have that $s^{\wedge}\langle v\rangle \square$ and $t^{\wedge}\langle y(0)\rangle$ are mixed by $a$. Additionally, by (b) of Lemma 2.8 we get that $s^{\wedge}\langle y(0)\rangle \uparrow$ and $s^{\wedge}\langle v\rangle \square$ are mixed by $a$, because $\min (v)=\min (y(0))$. Moreover since $\min (x(0))=\min (y(0))$, Lemma 2.13 yields that $s^{\wedge}\langle y(0)\rangle \uparrow$ and $t^{\wedge}\langle y(0)\rangle \uparrow$ are also mixed by $a$. Altogether, by transitivity of mixing we get that $t^{\wedge}\langle y(0)\rangle$ and $t^{\wedge}\langle y(0)\rangle \uparrow$ are mixed by $a$. But this contradicts case (c) of Lemma 2.11.

Lemma 2.27. Let $s, t \ll a$. Suppose $s$ and $t$ are mixed by a,s is min-separated by $a$ and $t$ is min-max-separated by $a$. Then $s^{\wedge} m \uparrow$ and $t^{\wedge} m \uparrow$ are mixed by a for every $m \ll a$.

Proof. Since $s$ and $t$ are mixed by $a$, there exist $x, y \ll a$ such that $\Delta\left(s^{\wedge} x\right)=\Delta\left(t^{\wedge} y\right)$. By Lemma 2.26 we must have that $\min (x(0))=\min (y(0))$. Hence Lemma 2.13 yields that $s^{\wedge} m \uparrow$ and $t^{\wedge} m \uparrow$ are mixed by $a$ for every $m \ll a$.

Lemma 2.28. Let $s, t \ll a$.

(a) Suppose $s \square$ and $t \square$ are mixed by $a$ and both $s \square$ and $t \square$ are still strongly separated by $a$. Then $s \square^{\wedge} m \square$ and $t \square^{\wedge} m \square$ are mixed by a for every $m \ll a$.

(b) Suppose $s \square$ and $t \square$ are mixed by $a, s \square$ is still strongly separated by $a$ and $t \square$ is very strongly separated by $a$. Then $s \square{ }^{\wedge} m \square$ and $t \square^{\wedge} m \uparrow$ are mixed 
by a for every $m \ll a$. Moreover $s \square^{\wedge} m \square$ and $t \square^{\wedge} m$ are separated by a for every $m \ll a$.

(c) Suppose $s \square$ and $t \square$ are mixed by $a$ and both $s \square$ and $t \square$ are very strongly separated by $a$. Then $s \square^{\wedge} m \uparrow$ and $t \square^{\wedge} m \uparrow$ are mixed by a for every $m \ll a$. Moreover $s \square^{\wedge} m$ and $t \square^{\wedge} m \uparrow$ are separated by a for every $m \ll a$.

Proof. Since $s \square$ and $t \square$ are mixed by $a$, there exist $x, y \ll a$ such that $\Delta(s \square \wedge x)=$ $\Delta(t \square \wedge y)$. In each of the three cases both $s \square$ and $t \square$ are strongly separated by $a$. Hence by Lemma 2.20 we must have that $x(0)$ is an initial segment of $y(0)$ or conversely. Since $\min (x(0))=\min (y(0))$, by Lemma 2.13 we have that $s \square^{\wedge} m \uparrow$ and $t \square^{\wedge} m \uparrow$ are mixed by $a$ for every $m \ll a$.

The rest of the result follows directly by the definition of being still and very strongly separated, using the transitivity of mixing.

Lemma 2.29. Let $s, t \ll a$. Suppose $s$ and $t \square$ are mixed by $a$ and $s$ is minseparated by $a$. Then $t \square$ is neither max-separated nor strongly separated by a.

Proof. Since $s$ and $t \square$ are mixed by $a$, there exist $x, y \ll a$ such that $\Delta\left(s^{\wedge} x\right)=$ $\Delta\left(t \square^{\wedge} y\right)$. Assume to the contrary that $t \square$ is either max-separated or strongly separated by $a$. Two applications of Lemma 2.12 yield that $\min (x(0))<\max (y(0))$ and $\max (x(0))>\min (y(0))$. We distinguish five cases.

For the first case suppose that $\min (x(0))<\min (y(0))$ and $\max (x(0))>\min (y(0))$. Let $v$ be the part of $x(0)$ below $\min (y(0))$. Since $\Delta\left(s^{\wedge} x\right)=\Delta\left(t \square^{\wedge} y\right)$, we have that $s^{\wedge}\langle v\rangle \uparrow$ and $t \square$ are mixed by $a$. Moreover $s$ and $t \square$ are also mixed by $a$. Hence by transitivity of mixing we obtain that $s^{\wedge}\langle v\rangle \uparrow$ and $s$ are mixed by $a$. But this contradicts case (a) of Lemma 2.11.

Next, assume that $t \square$ is max-separated by $a$ and $\min (x(0))>\min (y(0))$ as well as $\min (x(0))<\max (y(0))$. Choose $k$ with $\min (x(0)) \in a(k)$. Let $w$ denote the part of $y(0)$ less than or equal to $\max (a(k))$. If $\max (a(k))<\max (y(0))$, we get that $s^{\wedge}\langle a(k)\rangle \square$ and $t \square^{\wedge}\langle w\rangle \uparrow$ are mixed by $a$, because $\Delta\left(s^{\wedge} x\right)=\Delta\left(t \square^{\wedge} y\right)$. Otherwise, we must have that $\max (a(k))=\max (y(0))$, since $\min (x(0))<\max (y(0))$. Then we have that $s^{\wedge}\langle a(k)\rangle \square$ and $t \square^{\wedge}\langle y(0)\rangle$ are mixed by $a$. In the former case, by (c) of Lemma 2.8 we get that $t \square^{\wedge}\langle w\rangle \uparrow$ and $t \square$ are mixed by $a$. Moreover since $s$ and $t \square$ are mixed by $a$, by transitivity of mixing we obtain that $s^{\wedge}\langle a(k)\rangle \square$ and $s$ are mixed by $a$. This contradicts case (a) of Lemma 2.11. If we are in the latter case, we additionally have that $t \square^{\wedge}\langle y(0)\rangle$ and $t \square^{\wedge}\langle a(k)\rangle$ are mixed by $a$, because $t \square$ is max-separated by $a$ and $\max (a(k))=\max (y(0))$. By transitivity of mixing we can conclude that $s^{\wedge}\langle a(k)\rangle \square$ and $t \square^{\wedge}\langle a(k)\rangle$ are mixed by $a$. Moreover by (d) of canonical we must have that $s^{\wedge} m \square$ and $t \square^{\wedge} m$ are mixed by $a$ for all $m \ll a$. Finally, by (b) of Lemma 2.8 we have that $s^{\wedge} m \square$ and $s^{\wedge} n \square$ are mixed by $a$ for every $m, n \ll a$ with $\min (m)=\min (n)$. Again, by transitivity of mixing we obtain that $t \square^{\wedge} m$ and $t \square^{\wedge} n$ are mixed by $a$ for all $m, n \ll a$ with $\min (m)=\min (n)$. But this contradicts that $t \square$ is max-separated by $a$.

For the third case suppose that $t \square$ is strongly separated by $a$ and $\min (x(0))>$ $\min (y(0))$ as well as $\min (x(0))<\max (y(0))$. Let $v$ be the part of $y(0)$ below $\min (x(0))$. Since $\Delta\left(s^{\wedge} x\right)=\Delta\left(t \square^{\wedge} y\right)$, we have that $s$ and $t \square^{\wedge}\langle v\rangle \uparrow$ are mixed by $a$. Moreover $s$ and $t \square$ are mixed by $a$. Hence by transitivity of mixing we obtain that $t \square$ and $t \square^{\wedge}\langle v\rangle \uparrow$ are mixed by $a$. But this contradicts case (d) of Lemma 2.11

Now assume that $t \square$ is max-separated by $a$ and $\min (x(0))=\min (y(0))$. By Lemma 2.13 we get that $s^{\wedge} m \uparrow$ and $t \square^{\wedge} m \uparrow$ are mixed by $a$ for every $m \ll a$. 
Additionally, by (c) of Lemma 2.8 we have that $t \square^{\wedge} m \uparrow$ and $t \square$ are mixed by $a$ for all $m \ll a$, because $t \square$ is max-separated by $a$. Moreover since $s$ and $t \square$ are mixed by $a$, by transitivity of mixing we obtain that $s^{\wedge} m \uparrow$ and $s$ are mixed by $a$ for every $m \ll a$. This contradicts case (a) of Lemma 2.11 .

Finally, suppose that $t \square$ is strongly separated by $a$ and $\min (x(0))=\min (y(0))$. Equally, by Lemma 2.13 we get $s^{\wedge} m \uparrow$ and $t \square^{\wedge} m \uparrow$ are mixed by $a$ for every $m \ll a$. Additionally, by (b) of Lemma 2.8 we have that $s^{\wedge} m \uparrow$ and $s^{\wedge} n \uparrow$ are mixed by $a$ for all $m, n \ll a$ with $\min (m)=\min (n)$. Thus, by transitivity of mixing we get that $t \square^{\wedge} m \uparrow$ and $t \square^{\wedge} n \uparrow$ are mixed by $a$ for every $m, n \ll a$ with $\min (m)=\min (n)$. But this contradicts case (d) of Lemma 2.11

Lemma 2.30. Let $s, t \ll a$. Suppose $s \square$ and $t \square$ are mixed by a and $s \square$ is maxseparated by $a$. Then $t \square$ is neither min-max-separated nor strongly separated by $a$.

Proof. Since $s \square$ and $t \square$ are mixed by $a$, there exist $x, y \ll a$ such that $\Delta\left(s \square^{\wedge} x\right)=$ $\Delta\left(t \square^{\wedge} y\right)$. Assume to the contrary that $t \square$ is either min-max-separated or strongly separated by $a$. Two applications of Lemma 2.12 yield that $\max (x(0))>\min (y(0))$ and $\min (x(0))<\max (y(0))$. Now we distinguish three cases.

For the first case suppose that $\min (x(0))<\min (y(0))$ and $\max (x(0))>\min (y(0))$. Let $b$ with $a \ll b$ be as in (f) of canonical. Moreover let $v$ denote the part of $x(0)$ below $\min (y(0))$. Choose $k$ with $\min (y(0)) \in b(k)$. Additionally, let $w$ denote the part of $x(0)$ less than or equal to $\max (b(k))$. Since $\Delta\left(s \square^{\wedge} x\right)=\Delta\left(t \square^{\wedge} y\right)$, we have that $s \square^{\wedge}\langle v\rangle \uparrow$ and $t \square$ as well as $s \square^{\wedge}\langle w\rangle \uparrow$ and $t \square^{\wedge}\langle b(k)\rangle \uparrow$ are mixed by $b$. By (c) and (f) of canonical we have that $s \square$ is also max-separated by $b$. Therefore, by (c) of Lemma 2.8 we get that $s \square^{\wedge}\langle v\rangle \uparrow$ and $s \square^{\wedge}\langle w\rangle \uparrow$ are mixed by $b$. Thus, by transitivity of mixing we obtain that $t \square$ and $t \square^{\wedge}\langle w\rangle \uparrow$ are mixed by $b$. Now (d) and (f) of canonical yield that $t \square$ and $t \square^{\wedge} m \uparrow$ are mixed by $b$ for every $m \ll b$. Finally, since $a \ll b$, we can conclude that $t \square$ and $t \square^{\wedge} m \uparrow$ are mixed by $a$ for every $m \ll a$. But this contradicts cases (c) and (d) of Lemma 2.11

Next, suppose that $\min (x(0))>\min (y(0))$ and $\min (x(0))<\max (y(0))$. Let $v$ be the part of $y(0)$ below $\min (x(0))$. We have that $s \square$ and $t \square^{\wedge}\langle v\rangle \uparrow$ are mixed by $a$. Since $s \square$ and $t \square$ are mixed by $a$, we get that $t \square$ and $t \square^{\wedge}\langle v\rangle \uparrow$ are mixed by $a$, a contradiction as above.

Finally, assume that $\min (x(0))=\min (y(0))$. By Lemma 2.13 we have that $s \square^{\wedge} m \uparrow$ and $t \square^{\wedge} m \uparrow$ are mixed by $a$ for all $m \ll a$. Moreover by (c) of Lemma 2.8 we have that $s \square^{\wedge} m \uparrow$ and $s \square^{\wedge} n \uparrow$ are mixed by $a$ for every $m, n \ll a$, because $s \square$ is max-separated by $a$. By transitivity of mixing we get that $t \square^{\wedge} m \uparrow$ and $t \square^{\wedge} n \uparrow$ are mixed by $a$ for every $m, n \ll a$.

This contradicts cases (c) and (d) of Lemma 2.11

Lemma 2.31. Let $s, t \ll a$. Suppose $s$ and $t \square$ are mixed by $a$ and $s$ is min-maxseparated by $a$. Then $t \square$ is not strongly separated by $a$.

Proof. Since $s$ and $t \square$ are mixed by $a$, there exist $x, y \ll a$ such that $\Delta\left(s^{\wedge} x\right)=$ $\Delta\left(t \square^{\wedge} y\right)$. Assume to the contrary that $t \square$ is strongly separated by $a$. Two applications of Lemma 2.12 yield that $\max (x(0))>\min (y(0))$ and $\min (x(0))<\max (y(0))$. Now we distinguish three cases.

For the first case suppose that $\min (x(0))<\min (y(0))$ and $\max (x(0))>\min (y(0))$. Let $v$ be the part of $x(0)$ below $\min (y(0))$. Since $\Delta\left(s^{\wedge} x\right)=\Delta\left(t \square^{\wedge} y\right)$, we have that $s^{\wedge}\langle v\rangle \uparrow$ and $t \square$ are mixed by $a$. Moreover $s$ and $t \square$ are mixed by $a$. Hence by 
transitivity of mixing we get $s^{\wedge}\langle v\rangle \uparrow$ and $s$ are mixed by $a$. But this contradicts case (c) of Lemma 2.11.

Next, suppose that $\min (x(0))>\min (y(0))$ and $\min (x(0))<\max (y(0))$. Let $v$ be the part of $y(0)$ below $\min (x(0))$. Since $\Delta\left(s^{\wedge} x\right)=\Delta\left(t \square^{\wedge} y\right)$, we have that $s$ and $t \square^{\wedge}\langle v\rangle \uparrow$ are mixed by $a$. Moreover $s$ and $t \square$ are mixed by $a$. Hence by transitivity of mixing we get that $t \square$ and $t \square^{\wedge}\langle v\rangle \uparrow$ are mixed by $a$, contradicting case (d) of Lemma 2.11.

Finally, assume that $\min (x(0))=\min (y(0))$. By Lemma 2.13 we get that $s^{\wedge} m \uparrow$ and $t \square^{\wedge} m \uparrow$ are mixed by $a$ for all $m \ll a$. Moreover by (d) of Lemma 2.8 we have that $s^{\wedge} m \uparrow$ and $s^{\wedge} n \uparrow$ are mixed by $a$ for every $m, n \ll a$ with $\min (m)=\min (n)$, because $s$ is min-max-separated by $a$. By transitivity of mixing we get that $t \square^{\wedge} m \uparrow$ and $t \square^{\wedge} n \uparrow$ are mixed by $a$ for every $m, n \ll a$ with $\min (m)=\min (n)$. This contradicts case (d) of Lemma 2.11.

Now we define the parameter $\gamma$ of the mapping $\Gamma_{\gamma}$ which will canonize our given $\Delta$.

Definition. For given canonical $a$ define $\gamma:(a)^{<\omega} \rightarrow\{s m$, min-sep, max-sep, min-max, sss, vss $\}$ as follows: Let $\gamma(s)=s m$ iff $s$ is strongly mixed by $a$; moreover let $\gamma(s)=$ min-sep iff $s$ is min-separated by $a$; let $\gamma(s)=$ max-sep iff $s$ is maxseparated by $a$; let $\gamma(s)=$ min-max iff $s$ is min-max-separated by $a$; let $\gamma(s)=s s s$ iff $s$ is still strongly separated by $a$; finally, let $\gamma(s)=v s s$ iff $s$ is very strongly separated by $a$.

Recall that $\Gamma_{\gamma}$ is defined as follows:

For $m \in[\omega]^{<\omega}$ let $\operatorname{sm}(m)=\varnothing, \min -\operatorname{sep}(m)=\{\min (m)\}, \max -\operatorname{sep}(m)=$ $\{\max (m)\}, \min -\max (m)=\{\min (m), \max (m)\}$ and $s s s(m)=v s s(m)=m$.

Let $x \in(a)^{\omega}$. Define $\Gamma_{\gamma}(x)$ as follows: Let $k(0)=0$ and $\langle k(i): 0<i<N \leq \omega\rangle$ increasingly enumerate those $k$ such that $\gamma(x 1(k-1))=v s s$. Moreover let $k(N)=\omega$, if $N<\omega$. Now let $\Gamma_{\gamma}(x)=\left\langle\bigcup_{k(i) \leq j<k(i+1)} \gamma(x 1 j)(x(j)): i<N\right\rangle$.

Finally, we need three more definitions in order to give our last few lemmas.

Definition. Let $x, y \ll a$ and $k \in \omega$.

If possible, choose $i>0$ maximal such that $\max (x(i-1))<\min (a(k))$, otherwise choose $i=0$. Additionally, let $v$ denote the part of $x(i)$ below $\min (a(k))$. Now define $x_{k \downarrow}$ as follows: If $v=\varnothing$, let $x_{k \downarrow}=x 1 i$, otherwise let $x_{k \downarrow}=x 1 i^{\wedge}\langle v\rangle \uparrow$.

Next, choose $i$ minimal such that $\min (a(k)) \leq \min (x(i))$. Additionally if $i>0$, let $v$ denote the part of $x(i-1)$ larger than or equal to $\min (a(k))$, otherwise let $v=\varnothing$. Now define $x_{k \uparrow}$ as follows: If $v=\varnothing$, let $x_{k \uparrow}=x \uparrow i$, otherwise let $x_{k \uparrow}=\langle v\rangle^{\wedge} x\lceil i$.

Finally, if possible, choose $0<i<\operatorname{dom}\left(\Gamma_{\gamma}(x)\right)$, resp. $0<j<\operatorname{dom}\left(\Gamma_{\gamma}(y)\right)$, maximal such that $\max \left(\Gamma_{\gamma}(x)(i-1)\right)<\min (a(k))$, resp. $\max \left(\Gamma_{\gamma}(y)(j-1)\right)<$ $\min (a(k))$, otherwise choose $i=0$, resp. $j=0$. Additionally, let $v$, resp. $w$, denote the part of $\Gamma_{\gamma}(x)(i)$, resp. $\Gamma_{\gamma}(y)(j)$, below $\min (a(k))$. Now we say that $\Gamma_{\gamma}(x)$ corresponds with $\Gamma_{\gamma}(y)$ up to $k$ iff $\Gamma_{\gamma}(x) 1 i=\Gamma_{\gamma}(y) 1 j$ and $v=w$.

Remark. By definition of $x_{k \downarrow}$ and $x_{k \uparrow}$ it follows that $x_{k \downarrow}{ }^{\wedge} x_{k \uparrow}=x$ for every $x \ll a$ and $k \in \omega$.

Lemma 2.32. Let $x, y \ll a$. Suppose that $x_{i \downarrow}$ and $y_{i \downarrow}$ are mixed by a for every $i<\omega$. Then $\Delta(x)=\Delta(y)$. 
Proof. For every $i<\omega$ let $x_{i}, y_{i} \ll a$ be such that $\Delta\left(x_{i \downarrow}{ }^{\wedge} x_{i}\right)=\Delta\left(y_{i \downarrow}{ }^{\wedge} y_{i}\right)$. These sets exist, because $x_{i \downarrow}$ and $y_{i \downarrow}$ are mixed by $a$. Moreover by definition of $x_{k \downarrow}$ we obtain that $\lim _{i<\omega} x_{i \downarrow}{ }^{\wedge} x_{i}=x$ and $\lim _{i<\omega} y_{i \downarrow}{ }^{\wedge} y_{i}=y$. By (a) of canonical we have that $\Delta 1(a)^{\omega}$ is continuous. Hence we get that $\Delta(x)=\lim _{i<\omega} \Delta\left(x_{i \downarrow}{ }^{\wedge} x_{i}\right)$ and $\Delta(y)=\lim _{i<\omega} \Delta\left(y_{i \downarrow}{ }^{\wedge} y_{i}\right)$. Thus, $\lim _{i<\omega} \Delta\left(x_{i \downarrow}{ }^{\wedge} x_{i}\right)$ and $\lim _{i<\omega} \Delta\left(y_{i \downarrow} \wedge y_{i}\right)$ exist. Finally, since $\Delta\left(x_{i \downarrow} \wedge x_{i}\right)=\Delta\left(y_{i \downarrow} \wedge y_{i}\right)$ for every $i<\omega$, we get that $\lim _{i<\omega} \Delta\left(x_{i \downarrow} \wedge x_{i}\right)=$ $\lim _{i<\omega} \Delta\left(y_{i \downarrow} \wedge y_{i}\right)$, so we are done.

Lemma 2.33. Let $x, y \ll a$ and $k \in \omega$. Suppose that $\Gamma_{\gamma}(x)$ corresponds with $\Gamma_{\gamma}(y)$ up to $k$. Then $x_{k \downarrow}$ and $y_{k \downarrow}$ are mixed by a.

Proof. We prove the assertion in the lemma by induction on $k$.

Suppose first that $k=0$. By definition of $x_{k \downarrow}$ we have that $x_{0 \downarrow}=\varnothing$ and $y_{0 \downarrow}=\varnothing$. Thus, by definition of mixing we have that $x_{0 \downarrow}$ and $y_{0 \downarrow}$ are mixed by $a$.

Now assume that the assertion is true for some $k$. We show that it is also true for $k+1$. For that purpose suppose that $\Gamma_{\gamma}(x)$ corresponds with $\Gamma_{\gamma}(y)$ up to $k+1$. Hence $\Gamma_{\gamma}(x)$ also corresponds with $\Gamma_{\gamma}(y)$ up to $k$. By inductional assumption we have that $x_{k \downarrow}$ and $y_{k \downarrow}$ are mixed by $a$. Additionally, assume without loss of generality with $x_{k+1 \downarrow} \neq x_{k \downarrow}$ or $y_{k+1 \downarrow} \neq y_{k \downarrow}$. We distinguish ten cases.

For the first case suppose that both $x_{k \downarrow}$ and $y_{k \downarrow}$ are strongly mixed by $a$. We have that $s m(m)=\varnothing$ for every $m \ll a$. Since $\Gamma_{\gamma}(x)$ corresponds with $\Gamma_{\gamma}(y)$ up to $k+1$, we have that either $x_{k+1 \downarrow}=x_{k \downarrow}$ or $x_{k+1 \downarrow}=x_{k \downarrow} \wedge\langle a(k)\rangle \square$ and that either $y_{k+1 \downarrow}=y_{k \downarrow}$ or $y_{k+1 \downarrow}=y_{k \downarrow} \wedge^{\wedge}\langle a(k)\rangle \square$. By (a) of Lemma 2.8 we get that $x_{k \downarrow}$ and $x_{k \downarrow} \wedge\langle a(k)\rangle \square$ as well as $y_{k \downarrow}$ and $y_{k \downarrow}{ }^{\wedge}\langle a(k)\rangle \square$ are mixed by $a$. Moreover since $x_{k \downarrow}$ and $y_{k \downarrow}$ are mixed by $a$, by transitivity of mixing we obtain that $x_{k+1 \downarrow}$ and $y_{k+1 \downarrow}$ are mixed by $a$.

Next, assume that $x_{k \downarrow}$ is strongly mixed by $a$. Moreover suppose that $y_{k \downarrow}$ is either min-separated, min-max-separated or strongly separated by $a$. We have that $s m(m)=\varnothing$ as well as $\min -\operatorname{sep}(m)=\{\min (m)\}, \min -\max (m)=\{\min (m), \max (m)\}$ and $s s s(m)=v s s(m)=m$ for every $m \ll a$. Since $\Gamma_{\gamma}(x)$ corresponds with $\Gamma_{\gamma}(y)$ up to $k+1$, we must have that $x_{k+1 \downarrow}=x_{k \downarrow}{ }^{\wedge}\langle a(k)\rangle \square$ and $y_{k+1 \downarrow}=y_{k \downarrow}$. By (a) of Lemma 2.8 we get that $x_{k \downarrow}$ and $x_{k \downarrow}{ }^{\wedge}\langle a(k)\rangle \square$ are mixed by $a$. Therefore, since $x_{k \downarrow}$ and $y_{k \downarrow}$ are mixed by $a$, by transitivity of mixing we obtain that $x_{k+1 \downarrow}$ and $y_{k+1 \downarrow}$ are mixed by $a$.

Now assume that $x_{k \downarrow}$ is strongly mixed by $a$ and $y_{k \downarrow}$ is max-separated by $a$. We have that $\operatorname{sm}(m)=\varnothing$ and $\max -\operatorname{sep}(m)=\{\max (m)\}$ for every $m \ll a$. Since $\Gamma_{\gamma}(x)$ corresponds with $\Gamma_{\gamma}(y)$ up to $k+1$, we must have that either $x_{k+1 \downarrow}=x_{k \downarrow}$ or $x_{k+1 \downarrow}=x_{k \downarrow}{ }^{\wedge}\langle a(k)\rangle \square$ and that either $y_{k+1 \downarrow}=y_{k \downarrow}$ or $y_{k+1 \downarrow}=y_{k \downarrow}{ }^{\wedge}\langle a(k)\rangle \uparrow$. By (a) of Lemma 2.8 we get that $x_{k \downarrow}$ and $x_{k \downarrow} \wedge\langle a(k)\rangle \square$ are mixed by $a$. Moreover by (c) of Lemma 2.8 we get that $y_{k \downarrow}$ and $y_{k \downarrow}{ }^{\wedge}\langle a(k)\rangle \uparrow$ are mixed by $a$. Since $x_{k \downarrow}$ and $y_{k \downarrow}$ are mixed by $a$, by transitivity of mixing we obtain that $x_{k+1 \downarrow}$ and $y_{k+1 \downarrow}$ are mixed by $a$.

For the fourth case suppose that both $x_{k \downarrow}$ and $y_{k \downarrow}$ are min-separated by $a$. We have that $\min$-sep $(m)=\{\min (m)\}$ for every $m \ll a$. Since $\Gamma_{\gamma}(x)$ corresponds with $\Gamma_{\gamma}(y)$ up to $k+1$, we must have that $x_{k+1 \downarrow}=x_{k \downarrow}\langle a(k)\rangle \square$ and $y_{k+1 \downarrow}=y_{k \downarrow}{ }^{\wedge}\langle a(k)\rangle \square$. By Lemma 2.15 we get that $x_{k \downarrow}{ }^{\wedge}\langle a(k)\rangle$ and $y_{k \downarrow}{ }^{\wedge}\langle a(k)\rangle$ are mixed by $a$, because $x_{k \downarrow}$ and $y_{k \downarrow}$ are mixed by $a$. Moreover by (b) of Lemma 2.8 we get that $x_{k \downarrow} \wedge^{\wedge}\langle a(k)\rangle$ and $x_{k \downarrow}{ }^{\wedge}\langle a(k)\rangle \uparrow$ as well as $y_{k \downarrow}{ }^{\wedge}\langle a(k)\rangle$ and $y_{k \downarrow}{ }^{\wedge}\langle a(k)\rangle \uparrow$ are mixed by $a$. Therefore, possibly by transitivity of mixing, we obtain that $x_{k+1 \downarrow}$ and $y_{k+1 \downarrow}$ are mixed by $a$. 
Next, assume that $x_{k \downarrow}$ is min-separated by $a$ and $y_{k \downarrow}$ is min-max-separated by $a$. We have that $\min -\operatorname{sep}(m)=\{\min (m)\}$ and $\min -\max (m)=\{\min (m), \max (m)\}$ for every $m \ll a$. Since $\Gamma_{\gamma}(x)$ corresponds with $\Gamma_{\gamma}(y)$ up to $k+1$, we must have that $x_{k+1 \downarrow}=x_{k \downarrow}{ }^{\wedge}\langle a(k)\rangle \square$ and $y_{k+1 \downarrow}=y_{k \downarrow}{ }^{\wedge}\langle a(k)\rangle \uparrow$. By Lemma 2.27 we get that $\left.x_{k \downarrow} \wedge a(k)\right\rangle \uparrow$ and $y_{k \downarrow} \wedge\langle a(k)\rangle \uparrow$ are mixed by $a$, because $x_{k \downarrow}$ and $y_{k \downarrow}$ are mixed by $a$. Moreover by (b) of Lemma 2.8 we get that $x_{k \downarrow}{ }^{\wedge}\langle a(k)\rangle \uparrow$ and $x_{k \downarrow}{ }^{\wedge}\langle a(k)\rangle$ are mixed by $a$. Therefore, possibly by transitivity of mixing, we obtain that $x_{k+1 \downarrow}$ and $y_{k+1 \downarrow}$ are mixed by $a$.

We observe that if $x_{k \downarrow}$ is min-separated by $a$, then by Lemma $2.29 y_{k \downarrow}$ is neither max-separated nor strongly separated by $a$.

For the sixth case suppose that both $x_{k \downarrow}$ and $y_{k \downarrow}$ are max-separated by $a$. We have that $\max -\operatorname{sep}(m)=\{\max (m)\}$ for every $m \ll a$. Since $\Gamma_{\gamma}(x)$ corresponds with $\Gamma_{\gamma}(y)$ up to $k+1$, we must have that either $\left(\left(x_{k+1 \downarrow}=x_{k \downarrow} \vee x_{k+1 \downarrow}=x_{k \downarrow}{ }^{\wedge}\langle a(k)\rangle \uparrow\right)\right.$ $\left.\wedge\left(y_{k+1 \downarrow}=y_{k \downarrow} \vee y_{k+1 \downarrow}=y_{k \downarrow} \wedge\langle a(k)\rangle \uparrow\right)\right)$ or that $\left(x_{k+1 \downarrow}=x_{k \downarrow} \wedge\langle a(k)\rangle \wedge y_{k+1 \downarrow}=\right.$ $\left.y_{k \downarrow}{ }^{\wedge}\langle a(k)\rangle\right)$. By (c) of Lemma 2.8 we get that $x_{k \downarrow}$ and $x_{k \downarrow}{ }^{\wedge}\langle a(k)\rangle \uparrow$ as well as $y_{k \downarrow}$ and $y_{k \downarrow}{ }^{\wedge}\langle a(k)\rangle \uparrow$ are mixed by $a$. Moreover by Lemma 2.17 we get that $x_{k \downarrow}{ }^{\wedge}\langle a(k)\rangle$ and $y_{k \downarrow} \wedge\langle a(k)\rangle$ are mixed by $a$, because $x_{k \downarrow}$ and $y_{k \downarrow}$ are mixed by $a$. Therefore, possibly by transitivity of mixing, we obtain that $x_{k+1 \downarrow}$ and $y_{k+1 \downarrow}$ are mixed by $a$.

We observe that if $x_{k \downarrow}$ is max-separated by $a$, then by Lemma $2.30 y_{k \downarrow}$ is neither min-max-separated nor strongly separated by $a$.

For the seventh case assume that both $x_{k \downarrow}$ and $y_{k \downarrow}$ are min-max-separated by $a$. We have that $\min -\max (m)=\{\min (m), \max (m)\}$ for every $m \ll a$. Since $\Gamma_{\gamma}(x)$ corresponds with $\Gamma_{\gamma}(y)$ up to $k+1$, we must have that either $x_{k+1 \downarrow}=x_{k \downarrow}{ }^{\wedge}\langle a(k)\rangle \uparrow$ and $y_{k+1 \downarrow}=y_{k \downarrow}{ }^{\wedge}\langle a(k)\rangle \uparrow$ or that $x_{k+1 \downarrow}=x_{k \downarrow}{ }^{\wedge}\langle a(k)\rangle$ and $y_{k+1 \downarrow}=y_{k \downarrow}{ }^{\wedge}\langle a(k)\rangle$. By Lemma 2.19 we get that $x_{k \downarrow}{ }^{\wedge}\langle a(k)\rangle \uparrow$ and $y_{k \downarrow}{ }^{\wedge}\langle a(k)\rangle \uparrow$ as well as $x_{k \downarrow}{ }^{\wedge}\langle a(k)\rangle$ and $y_{k \downarrow} \wedge\langle a(k)\rangle$ are mixed by $a$, because $x_{k \downarrow}$ and $y_{k \downarrow}$ are mixed by $a$. Therefore, $x_{k+1 \downarrow}$ and $y_{k+1 \downarrow}$ are mixed by $a$.

We observe that if $x_{k \downarrow}$ is min-max-separated by $a$, then by Lemma $2.31 y_{k \downarrow}$ is not strongly separated by $a$.

For the eighth case suppose that both $x_{k \downarrow}$ and $y_{k \downarrow}$ are still strongly separated by $a$. We have that $s s s(m)=m$ for every $m \ll a$. Since $\Gamma_{\gamma}(x)$ corresponds with $\Gamma_{\gamma}(y)$ up to $k+1$, we must have that $x_{k+1 \downarrow}=x_{k \downarrow}{ }^{\wedge}\langle a(k)\rangle \square$ and $y_{k+1 \downarrow}=y_{k \downarrow}{ }^{\wedge}\langle a(k)\rangle \square$. By (a) of Lemma 2.28 we get that $x_{k \downarrow}{ }^{\wedge}\langle a(k)\rangle \square$ and $y_{k \downarrow}{ }^{\wedge}\langle a(k)\rangle \square$ are mixed by $a$, because $x_{k \downarrow}$ and $y_{k \downarrow}$ are mixed by $a$. Therefore, $x_{k+1 \downarrow}$ and $y_{k+1 \downarrow}$ are mixed by $a$.

Next, assume that $x_{k \downarrow}$ is still strongly separated by $a$ and $y_{k \downarrow}$ is very strongly separated by $a$. We have that $s s s(m)=v s s(m)=m$ for every $m \ll a$. Since $\Gamma_{\gamma}(x)$ corresponds with $\Gamma_{\gamma}(y)$ up to $k+1$, we must have that $x_{k+1 \downarrow}=x_{k \downarrow}{ }^{\wedge}\langle a(k)\rangle \square$ and $y_{k+1 \downarrow}=y_{k \downarrow}{ }^{\wedge}\langle a(k)\rangle \uparrow$. By (b) of Lemma 2.28 we get that $x_{k \downarrow}{ }^{\wedge}\langle a(k)\rangle \square$ and $y_{k \downarrow}{ }^{\wedge}\langle a(k)\rangle \uparrow$ are mixed by $a$, because $x_{k \downarrow}$ and $y_{k \downarrow}$ are mixed by $a$. Therefore, $x_{k+1 \downarrow}$ and $y_{k+1 \downarrow}$ are mixed by $a$.

Finally, suppose that both $x_{k \downarrow}$ and $y_{k \downarrow}$ are very strongly separated by $a$. We have that $\operatorname{vss}(m)=m$ for every $m \ll a$. Since $\Gamma_{\gamma}(x)$ corresponds with $\Gamma_{\gamma}(y)$ up to $k+1$, we must have that either $x_{k+1 \downarrow}=x_{k \downarrow} \wedge\langle a(k)\rangle \uparrow$ and $\left.y_{k+1 \downarrow}=y_{k \downarrow} \wedge a(k)\right\rangle \uparrow$ or that $x_{k+1 \downarrow}=x_{k \downarrow}{ }^{\wedge}\langle a(k)\rangle$ and $y_{k+1 \downarrow}=y_{k \downarrow}{ }^{\wedge}\langle a(k)\rangle$. By (c) of Lemma 2.28 we get that $x_{k \downarrow}{ }^{\wedge}\langle a(k)\rangle \uparrow$ and $y_{k \downarrow}{ }^{\wedge}\langle a(k)\rangle \uparrow$ are mixed by $a$, because $x_{k \downarrow}$ and $y_{k \downarrow}$ are mixed by $a$. Moreover by Lemma 2.23 we get that $x_{k \downarrow}{ }^{\wedge}\langle a(k)\rangle$ and $y_{k \downarrow}{ }^{\wedge}\langle a(k)\rangle$ are mixed by $a$. Therefore, $x_{k+1 \downarrow}$ and $y_{k+1 \downarrow}$ are mixed by $a$. 
Altogether, by symmetry we can conclude that in every case $x_{k+1 \downarrow}$ and $y_{k+1 \downarrow}$ are mixed by $a$. This completes the proof.

Lemma 2.34. Let $x, y \ll a$. Suppose that $\Gamma_{\gamma}(x)=\Gamma_{\gamma}(y)$. Then $\Delta(x)=\Delta(y)$.

Proof. First, we observe that $\Gamma_{\gamma}(x)$ corresponds with $\Gamma_{\gamma}(y)$ up to $i$ for every $i<\omega$, because $\Gamma_{\gamma}(x)=\Gamma_{\gamma}(y)$. Hence by Lemma 2.33 we get that $x_{i \downarrow}$ and $y_{i \downarrow}$ are mixed by $a$ for all $i<\omega$. Thus, Lemma 2.32 yields that $\Delta(x)=\Delta(y)$.

Lemma 2.35. Let $x, y \ll a$. Suppose that $\Gamma_{\gamma}(x) \neq \Gamma_{\gamma}(y)$. Then $\Delta(x) \neq \Delta(y)$.

Proof. Since $\Gamma_{\gamma}(x) \neq \Gamma_{\gamma}(y)$, we can choose $k$ maximal such that $\Gamma_{\gamma}(x)$ corresponds with $\Gamma_{\gamma}(y)$ up to $k$. By Lemma 2.33 we get that $x_{k \downarrow}$ and $y_{k \downarrow}$ are mixed by $a$. We show that $\Delta(x) \neq \Delta(y)$. For that purpose we distinguish nine cases.

For the first case assume that $x_{k \downarrow}$ is strongly mixed by $a$. Moreover suppose that $y_{k \downarrow}$ is either min-separated, min-max-separated or strongly separated by $a$. We have that $\operatorname{sm}(m)=\varnothing$ as well as $\min -\operatorname{sep}(m)=\{\min (m)\}, \min -\max (m)=$ $\{\min (m), \max (m)\}$ and $s s s(m)=v s s(m)=m$ for every $m \ll a$. Since $k$ is chosen maximal such that $\Gamma_{\gamma}(x)$ corresponds with $\Gamma_{\gamma}(y)$ up to $k$, we must have that either $x_{k+1 \downarrow}=x_{k \downarrow}$ or $x_{k+1 \downarrow}=x_{k \downarrow} \wedge\langle a(k)\rangle \square$ and that $y_{k+1 \downarrow}=y_{k \downarrow} \wedge\langle a(k)\rangle \square$. This implies that $\max \left(x_{k \uparrow}(0)\right)>\min \left(y_{k \uparrow}(0)\right)$. Thus, by Lemma 2.24 we obtain that $\Delta\left(x_{k \downarrow} \wedge x_{k \uparrow}\right) \neq \Delta\left(y_{k \downarrow} \wedge y_{k \uparrow}\right)$, because $x_{k \downarrow}$ and $y_{k \downarrow}$ are mixed by $a$.

Next, assume that $x_{k \downarrow}$ is strongly mixed by $a$ and $y_{k \downarrow}$ is max-separated by $a$. We have that $\operatorname{sm}(m)=\varnothing$ and $\max -\operatorname{sep}(m)=\{\max (m)\}$ for every $m \ll a$. Since $k$ is chosen maximal such that $\Gamma_{\gamma}(x)$ corresponds with $\Gamma_{\gamma}(y)$ up to $k$, we must have that either $x_{k+1 \downarrow}=x_{k \downarrow}$ or $x_{k+1 \downarrow}=x_{k \downarrow} \wedge\langle a(k)\rangle \square$ and that $y_{k+1 \downarrow}=y_{k \downarrow} \wedge\langle a(k)\rangle$. This implies that $\max \left(x_{k \uparrow}(0)\right) \geq \max \left(y_{k \uparrow}(0)\right)$. Thus, by Lemma 2.25] we obtain that $\Delta\left(x_{k \downarrow}{ }^{\wedge} x_{k \uparrow}\right) \neq \Delta\left(y_{k \downarrow} y_{k \uparrow}\right)$, because $x_{k \downarrow}$ and $y_{k \downarrow}$ are mixed by $a$.

We observe that we cannot have that both $x_{k \downarrow}$ and $y_{k \downarrow}$ are strongly mixed by $a$. This would contradict the choice of $k$, because $\operatorname{sm}(m)=\varnothing$ for all $m \ll a$.

For the third case suppose that both $x_{k \downarrow}$ and $y_{k \downarrow}$ are min-separated by $a$. We have that $\min$-sep $(m)=\{\min (m)\}$ for every $m \ll a$. Moreover we have chosen $k$ maximal such that $\Gamma_{\gamma}(x)$ corresponds with $\Gamma_{\gamma}(y)$ up to $k$. Therefore, by symmetry we must have without loss of generality that $x_{k+1 \downarrow}=x_{k \downarrow}$ and $y_{k+1 \downarrow}=y_{k \downarrow}\langle a(k)\rangle \square$. This implies that $\min \left(x_{k \uparrow}(0)\right)>\min \left(y_{k \uparrow}(0)\right)$. Thus, by Lemma 2.14 we obtain that $\Delta\left(x_{k \downarrow}{ }^{\wedge} x_{k \uparrow}\right) \neq \Delta\left(y_{k \downarrow}{ }^{\wedge} y_{k \uparrow}\right)$, because $x_{k \downarrow}$ and $y_{k \downarrow}$ are mixed by $a$.

Next, assume that $x_{k \downarrow}$ is min-separated by $a$ and $y_{k \downarrow}$ is min-max-separated by $a$. We have that $\min -\operatorname{sep}(m)=\{\min (m)\}$ and $\min -\max (m)=\{\min (m), \max (m)\}$ for every $m \ll a$. Since $k$ is chosen maximal such that $\Gamma_{\gamma}(x)$ corresponds with $\Gamma_{\gamma}(y)$ up to $k$, we must have that either $x_{k+1 \downarrow}=x_{k \downarrow} \wedge\langle a(k)\rangle \square$ and $y_{k+1 \downarrow}=y_{k \downarrow}, x_{k+1 \downarrow}=x_{k \downarrow}$ and $y_{k+1 \downarrow}=y_{k \downarrow} \wedge\langle a(k)\rangle \square$ or that $x_{k+1 \downarrow}=x_{k \downarrow} \wedge\langle a(k)\rangle \square$ and $y_{k+1 \downarrow}=y_{k \downarrow} \wedge\langle a(k)\rangle$. This implies that $\min \left(x_{k \uparrow}(0)\right) \neq \min \left(y_{k \uparrow}(0)\right)$ or $\max \left(x_{k \uparrow}(0)\right) \geq \max \left(y_{k \uparrow}(0)\right)$. Thus, by Lemma 2.26 we obtain that $\Delta\left(x_{k \downarrow}{ }^{\wedge} x_{k \uparrow}\right) \neq \Delta\left(y_{k \downarrow}{ }^{\wedge} y_{k \uparrow}\right)$, because $x_{k \downarrow}$ and $y_{k \downarrow}$ are mixed by $a$.

We observe that if $x_{k \downarrow}$ is min-separated by $a$, then by Lemma $2.29 y_{k \downarrow}$ is neither max-separated nor strongly separated by $a$.

For the fifth case suppose that both $x_{k \downarrow}$ and $y_{k \downarrow}$ are max-separated by $a$. We have that $\max -\operatorname{sep}(m)=\{\max (m)\}$ for every $m \ll a$. Moreover we have chosen $k$ maximal such that $\Gamma_{\gamma}(x)$ corresponds with $\Gamma_{\gamma}(y)$ up to $k$. Therefore, by symmetry we must have without loss of generality that either $x_{k+1 \downarrow}=x_{k \downarrow}$ or $x_{k+1 \downarrow}=x_{k \downarrow}\langle a(k)\rangle \uparrow$ and that $y_{k+1 \downarrow}=y_{k \downarrow}{ }^{\wedge}\langle a(k)\rangle$. This implies that $\max \left(x_{k \uparrow}(0)\right)>\max \left(y_{k \uparrow}(0)\right)$. Thus, 
by Lemma 2.16 we obtain that $\Delta\left(x_{k \downarrow} \wedge x_{k \uparrow}\right) \neq \Delta\left(y_{k \downarrow} \wedge y_{k \uparrow}\right)$, because $x_{k \downarrow}$ and $y_{k \downarrow}$ are mixed by $a$.

We observe that if $x_{k \downarrow}$ is max-separated by $a$, then by Lemma $2.30 y_{k \downarrow}$ is neither min-max-separated nor strongly separated by $a$.

For the sixth case assume that both $x_{k \downarrow}$ and $y_{k \downarrow}$ are min-max-separated by $a$. We have that $\min -\max (m)=\{\min (m), \max (m)\}$ for every $m \ll a$. Moreover we have chosen $k$ maximal such that $\Gamma_{\gamma}(x)$ corresponds with $\Gamma_{\gamma}(y)$ up to $k$. Therefore, by symmetry we must have without loss of generality that either $x_{k+1 \downarrow}=x_{k \downarrow}$ and $y_{k+1 \downarrow}=y_{k \downarrow}{ }^{\wedge}\langle a(k)\rangle \square$ or that $x_{k+1 \downarrow}=x_{k \downarrow}{ }^{\wedge}\langle a(k)\rangle \uparrow$ and $y_{k+1 \downarrow}=y_{k \downarrow} \wedge\langle a(k)\rangle$. This implies that $\min \left(x_{k \uparrow}(0)\right)>\min \left(y_{k \uparrow}(0)\right)$ or $\max \left(x_{k \uparrow}(0)\right)>\max \left(y_{k \uparrow}(0)\right)$. Thus, by Lemma 2.18 we obtain that $\Delta\left(x_{k \downarrow} x_{k \uparrow}\right) \neq \Delta\left(y_{k \downarrow} \wedge y_{k \uparrow}\right)$, because $x_{k \downarrow}$ and $y_{k \downarrow}$ are mixed by $a$.

We observe that if $x_{k \downarrow}$ is min-max-separated by $a$, then by Lemma $2.31 y_{k \downarrow}$ is not strongly separated by $a$.

For the seventh case suppose that both $x_{k \downarrow}$ and $y_{k \downarrow}$ are still strongly separated by $a$. We have that $s s s(m)=m$ for every $m \ll a$. Moreover we have chosen $k$ maximal such that $\Gamma_{\gamma}(x)$ corresponds with $\Gamma_{\gamma}(y)$ up to $k$. Therefore, by symmetry we must have without loss of generality that $x_{k+1 \downarrow}=x_{k \downarrow}$ and $y_{k+1 \downarrow}=y_{k \downarrow}{ }^{\wedge}\langle a(k)\rangle \square$. This implies that neither $x_{k \uparrow}(0)$ is an initial segment of $y_{k \uparrow}(0)$ nor conversely. Thus, by Lemma 2.20 we obtain that $\Delta\left(x_{k \downarrow} \wedge x_{k \uparrow}\right) \neq \Delta\left(y_{k \downarrow} y_{k \uparrow}\right)$, because $x_{k \downarrow}$ and $y_{k \downarrow}$ are mixed by $a$.

Next, assume that $x_{k \downarrow}$ is still strongly separated by $a$ and $y_{k \downarrow}$ is very strongly separated by $a$. We have that $s s s(m)=v s s(m)=m$ for every $m \ll a$. Since $k$ is chosen maximal such that $\Gamma_{\gamma}(x)$ corresponds with $\Gamma_{\gamma}(y)$ up to $k$, we must have that either $\left.x_{k+1 \downarrow}=x_{k \downarrow} \wedge a(k)\right\rangle \square$ and $y_{k+1 \downarrow}=y_{k \downarrow}, x_{k+1 \downarrow}=x_{k \downarrow}$ and $y_{k+1 \downarrow}=y_{k \downarrow}{ }^{\wedge}\langle a(k)\rangle \square$ or that $x_{k+1 \downarrow}=x_{k \downarrow} \wedge\langle a(k)\rangle \square$ and $y_{k+1 \downarrow}=y_{k \downarrow} \wedge\langle a(k)\rangle$. The former two cases imply that neither $x_{k \uparrow}(0)$ is an initial segment of $y_{k \uparrow}(0)$ nor conversely. Thus, by Lemma 2.20 we obtain that $\Delta\left(x_{k \downarrow} x_{k \uparrow}\right) \neq \Delta\left(y_{k \downarrow} y_{k \uparrow}\right)$, because $x_{k \downarrow}$ and $y_{k \downarrow}$ are mixed by $a$. In the latter case by (b) of Lemma 2.28 we get that $x_{k \downarrow}{ }^{\wedge}\langle a(k)\rangle \square$ and $y_{k \downarrow} \wedge\langle a(k)\rangle$ are separated by $a$. Therefore, by definition of separation we obtain that $\Delta\left(x_{k+1 \downarrow}{ }^{\wedge} x_{k+1 \uparrow}\right) \neq \Delta\left(y_{k+1 \downarrow}{ }^{\wedge} y_{k+1 \uparrow}\right)$.

Finally, suppose that both $x_{k \downarrow}$ and $y_{k \downarrow}$ are very strongly separated by $a$. We have that $\operatorname{vss}(m)=m$ for every $m \ll a$. Moreover we have chosen $k$ maximal such that $\Gamma_{\gamma}(x)$ corresponds with $\Gamma_{\gamma}(y)$ up to $k$. Therefore, by symmetry we must have without loss of generality that either $x_{k+1 \downarrow}=x_{k \downarrow} \wedge\langle a(k)\rangle \square$ and $y_{k+1 \downarrow}=y_{k \downarrow}$ or that $x_{k+1 \downarrow}=x_{k \downarrow}{ }^{\wedge}\langle a(k)\rangle \uparrow$ and $y_{k+1 \downarrow}=y_{k \downarrow}{ }^{\wedge}\langle a(k)\rangle$. This implies that $x_{k \uparrow}(0) \neq y_{k \uparrow}(0)$. Thus, by Lemma 2.22 we obtain that $\Delta\left(x_{k \downarrow}{ }^{\wedge} x_{k \uparrow}\right) \neq \Delta\left(y_{k \downarrow}{ }^{\wedge} y_{k \uparrow}\right)$, because $x_{k \downarrow}$ and $y_{k \downarrow}$ are mixed by $a$.

Altogether, by symmetry we can conclude that in every case $\Delta(x) \neq \Delta(y)$. This completes the proof.

Remark. Both the following definition and Lemma 2.36 are necessary to guarantee that property (b) of Theorem 0.7 follows from our Main Theorem.

Definition. Let $x, y \ll a$. We say that $\Gamma_{\gamma}(x)$ is a proper initial segment of $\Gamma_{\gamma}(y)$ iff there exists $k \in \omega$ such that $\Gamma_{\gamma}(x)$ corresponds with $\Gamma_{\gamma}(y)$ up to $k, x_{j \downarrow}$ is strongly mixed by $a$ for every $j \geq k$ and there exists $l \geq k$ such that $y_{l \downarrow}$ is separated in some sense by $a$. 
Lemma 2.36. There are no $x, y \ll$ a such that $\Gamma_{\gamma}(x)$ is a proper initial segment of $\Gamma_{\gamma}(y)$.

Proof. Assume to the contrary that there exist $x, y \ll a$ such that $\Gamma_{\gamma}(x)$ is a proper initial segment of $\Gamma_{\gamma}(y)$. According to the definition above there exists $k \in \omega$ such that $\Gamma_{\gamma}(x)$ corresponds with $\Gamma_{\gamma}(y)$ up to $k, x_{j \downarrow}$ is strongly mixed by $a$ for every $j \geq k$ and there exists $l \geq k$ such that $y_{l \downarrow}$ is separated in some sense by $a$. By Lemma 2.33 we get that $x_{k \downarrow}$ and $y_{k \downarrow}$ are mixed by $a$. Hence by definition of being mixed there exist $x_{0}, y_{0} \ll a$ such that $\Delta\left(x_{k \downarrow}{ }^{\wedge} x_{0}\right)=\Delta\left(y_{k \downarrow}{ }^{\wedge} y_{0}\right)$. Since $x_{j \downarrow}$ is strongly mixed by $a$ for all $j \geq k$, by (e) of canonical we have that $\left(x_{k \downarrow}{ }^{\wedge} x_{0}\right)_{j \downarrow}$ is also strongly mixed by $a$ for every $j \geq k$. Equally, since there exists $l \geq k$ such that $y_{l \downarrow}$ is separated in some sense by $a$, by (e) of canonical there exists $i \geq k$ such that $\left(y_{k \downarrow}{ }^{\wedge} y_{0}\right)_{i \downarrow}$ is also separated in some sense by $a$. Hence by definition of $\Gamma_{\gamma}$ we have $\Gamma_{\gamma}\left(x_{k \downarrow}{ }^{\wedge} x_{0}\right) \neq \Gamma_{\gamma}\left(y_{k \downarrow}{ }^{\wedge} y_{0}\right)$. Since $\Delta\left(x_{k \downarrow}{ }^{\wedge} x_{0}\right)=\Delta\left(y_{k \downarrow} \wedge y_{0}\right)$, we get a contradiction to Lemma 2.35.

This completes the proof of the lemma and with it the proof of the Main Theorem.

\section{Appendix. Corollaries}

Finally we show that both the theorem of Taylor (Theorem 0.4) and the theorem of Prömel-Voigt (Theorem 0.7) follow from our Main Theorem.

Proof (ad Theorem 0.4). Consider for a given $f:[\omega]^{<\omega} \rightarrow \omega$ the mapping $\Delta^{*}: \Omega^{\omega} \rightarrow$ $\omega$ with $x \mapsto f(x(0))$. First we show that $\Delta^{*}$ is a Borel measurable mapping, which is an assumption of our Main Theorem. Note that this result refers to the metric topology on $\Omega^{\omega}$. Therewith the sets $\prod_{i \in \omega} U_{i} \subseteq \Omega^{\omega}$ form a basis, where $U_{i}=[\omega]^{\omega}$ for all but finitely many $i \in \omega$. Since the inverse image $\left(\Delta^{*}\right)^{-1}$ of an arbitrary subset of $\omega$ is open, $\Delta^{*}$ is even continuous.

For all $k>0$ we have that $x 1 k$ is strongly mixed by every $a \ll \omega_{\max }$. This holds by definition of strongly mixing. Moreover $\varnothing$ cannot be still strongly separated by some $a$. Otherwise we would have that $\langle a(0)\rangle$ and $\langle a(0)\rangle \uparrow$ are mixed by $a$. By Corollary 2.21 we would get that $\langle a(0)\rangle \uparrow$ is still strongly separated by $a$, too. Because of our observation above we additionally have that $\langle a(0)\rangle$ is strongly mixed by $a$. Hence we would be in the case of Lemma 2.24 Since $\langle a(0)\rangle^{\wedge} x 1 k$ is still strongly mixed by $a$ for every $k$ and $x \ll a$, we would get a contradiction.

Invoking our Main Theorem there exist $\gamma^{*} \in\{s m$, min-sep, max-sep, min-max, $v s s\}$ and $a \in \Omega^{\omega}$ such that for all $x, y \ll a$

$$
f(x(0))=f(y(0)) \quad \text { iff } \quad \gamma^{*}(x(0))=\gamma^{*}(y(0)) .
$$

This is Theorem 0.4 .

Proof (ad Theorem 0.7). Assuming a Borel function $\Delta:[\omega]^{\omega} \rightarrow \mathbb{R}$ we construct the mapping $\Delta^{*}: \Omega^{\omega} \rightarrow \mathbb{R}$ with $x \mapsto \Delta(\{\min (x(i)): i \in \omega\})$. In order to apply our Main Theorem we have to prove that $\Delta^{*}$ is Borel. Let $g: \Omega^{\omega} \rightarrow[\omega]^{\omega}$ with $g(x)=\{\min (x(i)): i \in \omega\}$. Since $\Delta^{*}=\Delta \circ g$ and $\Delta$ is Borel, it is enough to show that $g$ is Borel. $\wp(\omega)$ can be identified with the Cantor space $2^{\omega}$ as a topological space endowed with the product topology. Since $[\omega]^{\omega} \subseteq \wp(\omega)$, for every $I, J \in[\omega]^{<\omega}$ with $I \cap J=\varnothing$ the sets $U_{I, J}=\left\{X \in[\omega]^{\omega}: \forall i \in I \forall j \in J i \in X \wedge j \notin X\right\}$ form a basis for the topology on $[\omega]^{\omega}$. It is obvious that the (sub)basis is countable, so it suffices to show that $g^{-1}\left(U_{I, J}\right)$ is Borel for each $I, J$. We have that $g^{-1}\left(U_{I, J}\right)=\{x \in$ 
$\left.\Omega^{\omega}:\{\min (x(i)): i \in \omega\} \in U_{I, J}\right\}$. The sets $\left\{\prod_{i \in \omega} V_{i}: \forall i \in \omega V_{i} \subseteq[\omega]^{<\omega} \wedge V_{i}=[\omega]^{<\omega}\right.$ for all but finitely many $i\} \cap \Omega^{\omega}$ form a basis for $\Omega^{\omega}$. Since only a finite number of pieces $x(i)$ consider the sets $I, J, g^{-1}\left(U_{I, J}\right)$ is a union of open sets of $\Omega^{\omega}$. Hence $g^{-1}\left(U_{I, J}\right)$ is open, too. Therewith $g$ is continuous. Since continuous mappings are Borel, $g$ is also Borel.

For given $a$ let $x \ll a$. Assume that for some $k$ the set $x 1 k$ is max-separated, min-max-separated or strongly separated by $a$. We have that $x 1 k^{\wedge}\langle x(k)\rangle$ and $x 1 k^{\wedge}\langle x(k) \cup x(k+1)\rangle$ are mixed by $a$, since $\langle x(k)\rangle$ and $\langle x(k) \cup x(k+1)\rangle$ have the same minimum and hence $\Delta^{*}\left(x 1 k^{\wedge}\langle x(k)\rangle^{\wedge} y\right)=\Delta^{*}\left(x 1 k^{\wedge}\langle x(k) \cup x(k+1)\rangle^{\wedge} y\right)$ for all $y \ll a$. But this contradicts the cases (b)-(d) of Lemma 2.11 Thus, for all $k$ we neither have the $x 1 k$ is max-separated, min-max-separated, still strongly separated nor very strongly separated by $a$.

Therewith our Main Theorem yields $\gamma^{*}: \Omega^{<\omega} \rightarrow\{$ sm, min-sep $\}$ and $a \in \Omega^{\omega}$ such that for all $x, y \ll a$ it holds that $\Delta^{*}(x)=\Delta^{*}(y)$ iff $\Gamma_{\gamma^{*}}(x)=\Gamma_{\gamma^{*}}(y)$. Let $A=\{\min (a(i)): i \in \omega\}$ and define for every $x \ll a$ the mapping $\Gamma:[A]^{\omega} \rightarrow[A] \leq \omega$ by $\Gamma(\{\min (x(i)): i \in \omega\}):=\Gamma_{\gamma^{*}}(x)$.

By definition of $\Gamma_{\gamma^{*}}$ we get that $\Gamma(X) \subseteq X$ for all $X \in[A]^{\omega}$.

Additionally, Theorem 0.7 requires that no $\Gamma(X)$ is a proper initial segment of some $\Gamma(Y)$. This property directly follows from Lemma 2.36.

Finally, since both $\Delta^{*}$ and $\Gamma_{\gamma^{*}}$ only depend on the minima of all pieces, for all $X, Y \in[A]^{\omega}$ it follows that $\Delta(X)=\Delta(Y)$ iff $\Gamma(X)=\Gamma(Y)$.

\section{REFERENCES}

[Ra30] F. P. Ramsey, On a problem of formal logic, Proceedings of the London Mathematical Society (2), vol. 30 (1930), pp. 264-286.

[ErRa50] P. Erdös and R. Rado, A combinatorial theorem, J. London Math. Soc., vol. 25 (1950), pp. 249-255. MR0037886(12:322f)

[Ku66] K. Kuratowski, Topology, Academic Press, New York, vol. 1 (1966). MR0217751 $(36: 840)$

[Ma68] A. R. D. Mathias, On a generalization of Ramsey's theorems, Notices of the American Mathematical Society, vol. 15 (1968), p. 931.

[Si70] J. Silver, Every analytic set is Ramsey, The Journal of Symbolic Logic, vol. 35 (1970), no. 1, pp. 60-64. MR0332480 (48:10807)

[GrRo71] R. L. Graham and B. L. Rothschild, Ramsey's theorem for n-parameter sets, Trans. Amer. Math. Soc., vol. 159 (1971), pp. 413-432. MR0284352 (44:1580)

[GaPr73] F. Galvin and K. Prikry, Borel sets and Ramsey's Theorem, The Journal of Symbolic Logic, vol. 38 (1973), no. 2, pp. 193-198. MR0337630 (49:2399)

[Ba74] J. E. Baumgartner, A short proof of Hindman's Theorem, Journal of Combinatorial Theory (A), vol. 17 (1974), pp. 384-386. MR0354394 (50:6873)

[El74] E. Ellentuck, A new proof that analytic sets are Ramsey, The Journal of Symbolic Logic, vol. 39 (1974), no. 1, pp. 163-165. MR0349393 (50:1887)

[Hi74] N. Hindman, Finite sums from sequences within cells of a partition of $N$, Journal of Combinatorial Theory (A), vol. 17 (1974), pp. 1-11. MR0349574 (50:2067)

[Mi75] K. R. Milliken, Ramsey's Theorem with Sums or Unions, Journal of Combinatorial Theory (A), vol. 18 (1975), pp. 276-290. MR0373906 (51:10106)

[Ta76] A. D. Taylor, A Canonical Partition Relation for Finite Subsets of $\omega$, Journal of Combinatorial Theory (A), vol. 21 (1976), pp. 137-146. MR0424571 (54:12530)

[Ma77] A. R. D. Mathias, Happy families, Annals of Math. Logic, vol. 12 (1977), pp. 59-111. MR 0491197 (58:10462)

[PuRö82] P. Pudlak and V. Rödl, Partition theorems for systems of finite subsets of integers, Discrete Math., vol. 39 (1982), pp. 67-73. MR0677888 (84i:05018) 
[PrVo85] H. J. Prömel and B. Voigt, Canonical Forms of Borel-Measurable Mappings $\Delta:[\omega]]^{\omega} \rightarrow$ $\mathbb{R}$, Journal of Combinatorial Theory (A), vol. 40 (1985), pp. 409-417. MR0814423 (87g:04004)

[Ke95] A. S. Kechris, Classical Descriptive Set Theory, Springer-Verlag, Berlin, 1995. MR.1321597 (96e:03057)

[To98] S. Todorcevic, Infinite-Dimensional Ramsey Theory, Preprint, (1998).

[Sp01] O. Spinas, Canonical behaviour of Borel functions on superperfect rectangles, Journal of Math. Logic, vol. 1, no. 2 (2001), pp. 173-220. MR.1864736 (2002h:03107)

Mathematisches Seminar, Christian-Albrechts-Universität Zu Kiel, Ludewig-MeynStrasse 4, 24098 Kiel, Germany

E-mail address: olaf.klein@teacheries.com

Mathematisches Seminar, Christian-Albrechts-Universität Zu Kiel, Ludewig-MeynStrasse 4, 24098 Kiel, Germany

E-mail address: spinas@math.uni-kiel.de 